\title{
HOPF-BIFURCATION THEOREM AND STABILITY FOR THE MAGNETO-HYDRODYNAMICS EQUATIONS
}

\author{
WEIPING YAN
}

\begin{abstract}
This paper is devoted to the study of the dynamical behavior for the 3D viscous Magneto-hydrodynamics equations. We first prove that this system under smooth external forces possesses time dependent periodic solutions, bifurcating from a steady solution. If the time periodic solution is smooth, then the linear stability of the time periodic solution implies nonlinear stability is obtained in $\mathbf{L}^{p}$ for all $p \in(3, \infty)$
\end{abstract}

\section{Introduction and main results}

We consider the 3D incompressible magneto-hydrodynamics (MHD) equations under external time-independent force

$$
\begin{aligned}
U_{t}-\nu \triangle U+(U \cdot \nabla) U & =-\nabla P-\frac{1}{2} \nabla H^{2}+H \cdot \nabla H+f_{\alpha}, \\
H_{t}-\eta \triangle H+(U \cdot \nabla) H & =H \cdot \nabla U+h_{\alpha}, \\
\nabla \cdot H & =\nabla \cdot U=0,
\end{aligned}
$$

where $U$ is the flow velocity vector, $H$ is the magnetic field vector, the kinematic viscosity $\nu$ and the magnetic diffusivity $\kappa$ are positive constants. $P$ is a scalar pressure, $f_{\alpha}$ and $h_{\alpha}$ are external time independent forces, which depend smoothly on some parameter $\alpha$.

2010 Mathematics Subject Classification. 76W05, 35B10, 76E25.

Key words and phrases. Magneto-hydrodynamics equations, periodic solution, Hopf bifurcation, stability. 
Since the work of Sattinger [19], Iudovich [14] and Iooss [11] in 1971, the bifurcation of periodic solutions from stationary solutions (i.e. Hopf-bifurcation) of incompressible Navier-Stokes equation has attracted much attention, see [2], [9], [12], [13], etc. When the linearized operator possesses a continuous spectrum up to the imaginary axis and that a pair of imaginary eigenvalues crosses the imaginary axis, A. Melcher et al. [17] proved Hopf-bifurcation for the vorticity formulation of the incompressible Navier-Stokes equations in $\mathbb{R}^{3}$. Their work is mainly motivated by the work of T. Brand et al. [1] who studied the Hopfbifurcation problem and its exchange of stability for a coupled reaction diffusion model in $\mathbb{R}^{a}$. We mention that Crandall and Rabinowitz [4] gave an abstract infinite-dimensional version of Hopf bifurcation theorem which has found many applications. But we can not directly use the method of dealing with NavierStokes equation to magneto-hydrodynamics equations because of the presence of the magnetic field and its interaction with the hydrodynamic motion in the MHD flow of large oscillation. In this paper, our aim is first to establish the corresponding Hopf-bifurcation result for the three-dimensional magneto-hydrodynamics equations. Then, we prove that if the time periodic solution is smooth, then it is $\left(\mathbf{L}^{q}, \mathbf{L}^{q}\right)$ nonlinearly stable in the sense of Lyapunov.

By [20], we know that external forces $f_{\alpha}$ and $h_{\alpha}$ can be chosen suitably so that $\left(U_{\alpha}(x)+U_{c_{1}}, H_{\alpha}(x)+H_{c_{1}}, P_{\alpha}(x)\right)$ is the solution of the steady magnetohydrodynamics equation

$$
\begin{aligned}
-\nu \triangle U+(U \cdot \nabla) U & =-\nabla P-\frac{1}{2} \nabla H^{2}+H \cdot \nabla H+f_{\alpha}, \\
-\eta \triangle H+(U \cdot \nabla) H & =H \cdot \nabla U+h_{\alpha}, \\
\nabla \cdot H & =\nabla \cdot U=0,
\end{aligned}
$$

with $U_{c}=\left(c_{1}, 0,0\right)^{T}, H_{c}=\left(c_{1}, 0,0\right)^{T}$ and

$$
\lim _{|x| \rightarrow \infty} U_{\alpha}(x)=\mathbf{0}, \quad \lim _{|x| \rightarrow \infty} H_{\alpha}(x)=\mathbf{0},
$$

where $\mathbf{0}=(0,0,0)^{T}$.

To seek the periodic solution, we linearize system (1.1)-(1.2) about the steady state $\left(U_{\alpha}+U_{c_{1}}, H_{\alpha}+H_{c_{1}}, P_{\alpha}\right)$ by writing

$$
U=u+U_{\alpha}+U_{c_{1}}, \quad H=v+H_{\alpha}+H_{c_{1}}, \quad p=P-P_{\alpha} .
$$

Then, the deviation $(u, v, p)$ from the stationary $\left(U_{\alpha}+U_{c_{1}}, H_{\alpha}+H_{c_{1}}, P_{\alpha}\right)$ satisfies

$$
\begin{aligned}
u_{t}-\nu & \Delta u+c_{1} \partial_{x_{1}} u+u_{\alpha} \cdot \nabla u+u \cdot \nabla u_{\alpha}+u \cdot \nabla u \\
= & -\nabla p-\frac{1}{2} \nabla\left(\left|H_{\alpha}+v\right|^{2}-\left|H_{\alpha}\right|^{2}\right)+H_{\alpha} \cdot \nabla u+u \cdot \nabla H_{\alpha}+v \cdot \nabla v
\end{aligned}
$$


(1.8) $v_{t}-\eta \Delta v+c_{1} \partial_{x_{2}} v+u_{\alpha} \cdot \nabla v+u \cdot \nabla v_{\alpha}+u \cdot \nabla v$

$$
=v_{\alpha} \cdot \nabla u+v \cdot \nabla u_{\alpha}+v \cdot \nabla u,
$$

with incompressible condition

$$
\nabla \cdot u=\nabla \cdot v=0 .
$$

Here, for general matrices $u=\left(u_{i j}\right)_{i, j=1,2,3}$,

$$
\nabla \cdot u=\left(\sum_{j=1}^{3} \partial_{x_{1}} u_{1 j}, \sum_{j=1}^{3} \partial_{x_{1}} u_{2 j}, \sum_{j=1}^{3} \partial_{x_{1}} u_{3 j}\right)^{T} .
$$

In fact, by the incompressible condition (1.9), it follows that

$$
\nabla \cdot\left(u v^{T}\right)=u \cdot \nabla u+u \nabla \cdot u=u \cdot \nabla u .
$$

Thus using (1.9) and (1.10) to (1.7)-(1.8), we obtain

$$
\begin{gathered}
\text { (1.11) } \begin{aligned}
u_{t}-\nu \Delta u+c_{1} \partial_{x_{1}} u+\nabla \cdot\left(u_{\alpha} u^{T}\right) & +\nabla \cdot\left(u u_{\alpha}^{T}\right)+\nabla \cdot\left(u u^{T}\right) \\
=-\nabla p-\frac{1}{2} \nabla\left(\left|H_{\alpha}+v\right|^{2}-\left|H_{\alpha}\right|^{2}\right) & \\
+ & \nabla \cdot\left(H_{\alpha} u^{T}\right)+\nabla \cdot\left(u H_{\alpha}^{T}\right)+\nabla \cdot\left(v v^{T}\right), \\
(1.12) \quad v_{t}-\eta \Delta v+c_{1} \partial_{x_{1}} v+\nabla \cdot\left(u_{\alpha} v^{T}\right) & +\nabla \cdot\left(u v_{\alpha}^{T}\right)+\nabla \cdot\left(u v^{T}\right) \\
& =\nabla \cdot\left(v_{\alpha} u^{T}\right)+v \cdot \nabla u_{\alpha}+\nabla \cdot\left(v u^{T}\right) .
\end{aligned}
\end{gathered}
$$

The vorticity associated with velocity field $u$ of the fluid is defined by $\omega=\nabla \times u$. Then, using $\nabla \times \nabla \cdot\left(u u^{T}\right)=\nabla \cdot\left(\omega u^{T}-u \omega^{T}\right)$, we can rewrite system (1.11) as

$$
\begin{gathered}
\omega_{t}-\nu \Delta \omega+c_{1} \partial_{x_{1}} \omega+\nabla \cdot\left(\omega_{\alpha} u^{T}-u_{\alpha} \omega^{T}\right) \\
\quad+\nabla \cdot\left(\omega u_{\alpha}^{T}-u \omega_{\alpha}^{T}\right)+\nabla \cdot\left(\omega u^{T}-u \omega^{T}\right) \\
=-\frac{1}{2} \nabla \times \nabla\left(\left|H_{\alpha}+v\right|^{2}-\left|H_{\alpha}\right|^{2}\right)+\nabla \cdot\left(\nabla \times H_{\alpha} u^{T}-H_{\alpha} \omega^{T}\right) \\
\quad+\nabla \cdot\left(\omega H_{\alpha}^{T}-u \nabla \times H_{\alpha}^{T}\right)+\nabla \cdot\left(\nabla \times v v^{T}-v \nabla \times v^{T}\right) .
\end{gathered}
$$

Note that the space of divergence free vector fields is invariant under the evolution (1.13). We can assume that $\nabla \cdot \omega=0$. Moreover, we can reconstruct the velocity $u$ from the vorticity $\omega$ by solving the equation

$$
\nabla \times u=\omega, \quad \nabla \cdot \omega=0 .
$$

Denote $\varphi=(\omega, v)^{T}$. Then, we can write system (1.12)-(1.13) as the evolution equation form

$$
\frac{d \varphi}{d t}+\mathcal{N} \varphi+G(\varphi)=F(\varphi)
$$


where

$$
\mathcal{N}=\left(\begin{array}{cc}
-\nu \triangle+c_{1} \partial_{x_{1}} & 0 \\
0 & -\eta \triangle+c_{1} \partial_{x_{1}}
\end{array}\right)
$$

and

$$
G(\varphi)=\left(\begin{array}{c}
g^{1} \\
g^{2}
\end{array}\right), \quad F(\varphi)=\left(\begin{array}{c}
g^{3} \\
g^{4}
\end{array}\right)
$$

with

$$
\begin{aligned}
g^{1}= & \nabla \cdot\left(\omega_{\alpha} u^{T}-u_{\alpha} \omega^{T}\right)+\nabla \cdot\left(\omega u_{\alpha}^{T}-u \omega_{\alpha}^{T}\right)+\nabla \times \nabla\left(H_{\alpha} v\right) \\
& -\nabla \cdot\left(\nabla \times H_{\alpha} u^{T}-H_{\alpha} \omega^{T}\right)-\nabla \cdot\left(\omega H_{\alpha}^{T}-u \nabla \times H_{\alpha}^{T}\right), \\
g^{2}= & \nabla \cdot\left(u_{\alpha} v^{T}\right)+\nabla \cdot\left(u v_{\alpha}^{T}\right)-\nabla \cdot\left(v_{\alpha} u^{T}\right)-v \cdot \nabla u_{\alpha}, \\
g^{3}= & -\nabla \cdot\left(\omega u^{T}-u \omega^{T}\right)-\frac{1}{2} \nabla \times \nabla\left(\left|H_{\alpha}+v\right|^{2}-\left|H_{\alpha}\right|^{2}-2 H_{\alpha} v\right) \\
& +\nabla \cdot\left(\nabla \times v v^{T}-v \nabla \times v^{T}\right), \\
g^{4}= & \nabla \cdot\left(v u^{T}\right)-\nabla \cdot\left(u v^{T}\right) .
\end{aligned}
$$

We denote $\widehat{G}_{\alpha}$ by $\widehat{G}$ for convenience. One overcomes usually the problem of the essential spectrum of operator $-(\widehat{\mathcal{N}}+\widehat{G})$ up to the imaginary axis, we need the following assumption:

(H1) For any $\alpha \in\left[\alpha_{c}-\alpha_{0}, \alpha_{c}+\alpha_{0}\right],(0,0)$ is not an eigenvalue of $\widehat{\mathcal{N}}+\widehat{G}$.

(H2) For $\alpha=\alpha_{c}$, the operator $-(\widehat{\mathcal{N}}+\widehat{G})$ has two pair eigenvalues $\left(\lambda_{0}^{+}, \mu_{0}^{+}\right)$ and $\left(\lambda_{0}^{-}, \mu_{0}^{-}\right)$satisfying

$$
\begin{gathered}
\lambda_{0}^{ \pm}\left(\alpha_{c}\right)=\mu_{0}^{ \pm}\left(\alpha_{c}\right)= \pm i \xi_{0} \neq 0, \quad \text { for } \xi_{0}>0, \\
\left.\frac{d}{d \alpha} \operatorname{Re}\left(\lambda_{0}^{ \pm}(\alpha)\right)\right|_{\alpha=\alpha_{c}},\left.\quad \frac{d}{d \alpha} \operatorname{Re}\left(\mu_{0}^{ \pm}(\alpha)\right)\right|_{\alpha=\alpha_{c}}>0 .
\end{gathered}
$$

(H3) The rest eigenvalue of $-(\widehat{\mathcal{N}}+\widehat{G})$ is strictly bounded away from the imaginary axis in the left half plane for all $\alpha \in\left[\alpha_{c}-\alpha_{0}, \alpha_{c}+\alpha_{0}\right]$.

Under the generic assumption the cubic coefficient terms $a_{1}, a_{2} \neq 0$ in (3.41)(3.42), Hopf-bifurcation result about MHD is stated:

TheOrem 1.1. Assume that (H1)-(H3) hold. Then system (1.1)-(1.3) has a one dimensional family of small time-periodic solutions, i.e.

$$
U(x, t)=U\left(x, t+2 \pi / \xi_{1}\right), \quad H(x, t)=H\left(x, t+2 \pi / \xi_{2}\right)
$$

with $\alpha=\alpha_{c}+\varepsilon, \varepsilon \in\left(0, \alpha_{0}\right)$. Moreover, $\xi_{1}=\xi_{0}+\mathcal{O}(\varepsilon), \xi_{2}=\xi_{0}+\mathcal{O}(\varepsilon)$, and

$$
\|U\|_{C_{b}^{0}\left(\mathbb{R}^{3} \times\left[0,2 \pi / \xi_{1}\right]\right)}=\mathcal{O}(\varepsilon), \quad\|H\|_{C_{b}^{0}\left(\mathbb{R}^{3} \times\left[0,2 \pi / \xi_{2}\right]\right)}=\mathcal{O}(\varepsilon) .
$$

Now we give the definition of Lyapunov stability and instability in the framework MHD. This definition is a small modification of Definition 2.1 in [21]. 
Definition 1.2. Let $(X, Z)$ be a pair of Banach spaces. An equilibrium $\left(u_{\alpha}, H_{\alpha}\right)$ which is the solution of (1.4)-(1.6) is called $(X, Z)$ Lyapunov nonlinearly stable if, no matter how small $\varepsilon>0$, there exist $\sigma>0$ and $\left(u_{0}, v_{0}\right) \in X$ such that $\left\|\left(u_{0}, v_{0}\right)\right\|_{Z}<\sigma$ imply the following two assertions:

(a) there exists a global in time solution to (1.11)-(1.12) such that $(u, v) \in$ $\mathbf{C}([0, \infty) ; X)$;

(b) $\|(u, v)\|_{Z} \leq \varepsilon$ for almost every $t \in[0, \infty)$.

An equilibrium $\left(U_{\alpha}, H_{\alpha}\right)$ that is unstable in the above sense is called Lyapunov nonlinearly unstable.

Theorem 1.3. Let $q>3$. Assume that (H2) holds. If MHD (1.1)-(1.3) has a smooth time periodic solution $(U, H)$, then $(U, H)$ is $\left(\boldsymbol{L}^{q}, \boldsymbol{L}^{q}\right)$ nonlinearly stable in the sense of Lyapunov.

This paper is organized as follows. In Section 2, we introduce some notation and preliminaries. In section 3, the main proof of Theorem 1.1 is carried out by using Lyapunov-Schmidt method. In section 4, using a bootstrap argument, we prove that the linear stability of time periodic solution implies nonlinear instability for MHD (1.1)-(1.3).

\section{Preliminaries}

We start this section by introducing some notations. Consider the following standard Sobolev space, spatially weighted Lebesgue space

$$
\begin{aligned}
\mathbf{W}_{\kappa}^{q} & :=\left\{u:\|u\|_{\kappa}^{q}:=\sum_{|\alpha| \leq \kappa}\left\|D^{\alpha} u\right\|_{\mathbf{L}^{q}}^{q}<\infty\right\}, \\
\mathbf{L}_{s}^{p} & :=\left\{u:\|u\|_{s}^{p}:=\int_{\mathbb{R}^{3}} \rho^{s}(x) u^{p}(x) d x<\infty\right\},
\end{aligned}
$$

where weighted function $\rho(x)=\sqrt{1+|x|^{2}}$. The Fourier transform is a continuous mapping from $\mathbf{L}_{s}^{p}$ into $\mathbf{W}_{\kappa}^{q}$. Especially, when $p=2$, the Fourier transform is an isomorphism between $\mathbf{H}^{p}$ and $\mathbf{L}_{p}^{2}$ with $\|u\|_{\mathbf{L}_{p}^{2}}=\left\|\rho^{p} u\right\|_{\mathbf{L}^{2}}$.

To investigate periodic solutions of system (1.1)-(1.2), we also introduce the space $\mathbf{X}:=\left\{u=\left(u_{n}\right)_{n \in \mathbf{Z}}:\|u\|_{\mathbf{X}}<\infty\right\}$ and weighted space $\mathcal{L}_{s}^{p}=\mathbf{L}_{s}^{p} \times \mathbf{L}_{s}^{p}$, $\mathcal{H}^{m}=\mathbf{H}^{m} \times \mathbf{H}^{m}, \mathcal{X}=\mathbf{X} \times \mathbf{X}$, with norms

$$
\begin{aligned}
\|u\|_{\mathbf{X}} & =\sum_{n \in \mathbf{Z}}\left\|u_{n}\right\|_{\mathbf{H}^{p}}, \quad\|\varphi\|_{\mathcal{X}}:=\|u\|_{\mathbf{X}}+\|v\|_{\mathbf{X}}, \\
\|\widehat{\varphi}\|_{\mathcal{L}_{s}^{p}}:=\|\widehat{u}\|_{\mathbf{L}_{s}^{p}}+\|\widehat{v}\|_{\mathbf{L}_{s+1}^{p}}, & \|\widehat{\varphi}\|_{\mathcal{H}^{m}}:=\|u\|_{\mathbf{H}^{m}}+\|v\|_{\mathbf{H}^{m}},
\end{aligned}
$$

for $\varphi=(u, v)^{T} \in \mathcal{L}_{s}^{p}$ or $\mathcal{X}$, respectively.

In this paper, we consider the following form of time-periodic solution

$$
\omega=\omega\left(x, t / \xi_{1}\right), \quad v=v\left(x, t / \xi_{2}\right),
$$


where $\xi_{1}, \xi_{2} \in \mathbf{R}^{+}$denote the corresponding frequencies.

Thus we need to find $2 \pi$ time periodic solutions of

$$
\Xi \frac{d \varphi}{d t}+\mathcal{N} \varphi+G(\varphi)=F(\varphi)
$$

where

$$
\Xi=\left(\begin{array}{cc}
\xi_{1} & 0 \\
0 & \xi_{2}
\end{array}\right), \quad \mathcal{N}=\left(\begin{array}{cc}
-\nu \triangle+c_{1} \partial_{x_{1}} & 0 \\
0 & -\eta \triangle+c_{1} \partial_{x_{1}}
\end{array}\right),
$$

and

with

$$
G(\varphi)=\left(\begin{array}{c}
g^{1} \\
g^{2}
\end{array}\right), \quad F(\varphi)=\left(\begin{array}{c}
g^{3} \\
g^{4}
\end{array}\right)
$$

$$
\begin{aligned}
g^{1}= & \nabla \cdot\left(\omega_{\alpha} u^{T}-u_{\alpha} \omega^{T}\right)+\nabla \cdot\left(\omega u_{\alpha}^{T}-u \omega_{\alpha}^{T}\right)+\nabla \times \nabla\left(H_{\alpha} v\right) \\
& -\nabla \cdot\left(\nabla \times H_{\alpha} u^{T}-H_{\alpha} \omega^{T}\right)-\nabla \cdot\left(\omega H_{\alpha}^{T}-u \nabla \times H_{\alpha}^{T}\right), \\
g^{2}= & \nabla \cdot\left(u_{\alpha} v^{T}\right)+\nabla \cdot\left(u v_{\alpha}^{T}\right)-\nabla \cdot\left(v_{\alpha} u^{T}\right)-v \cdot \nabla u_{\alpha}, \\
g^{3}= & -\nabla \cdot\left(\omega u^{T}-u \omega^{T}\right)-\frac{1}{2} \nabla \times \nabla\left(\left|H_{\alpha}+v\right|^{2}-\left|H_{\alpha}\right|^{2}-2 H_{\alpha} v\right) \\
& +\nabla \cdot\left(\nabla \times v v^{T}-v \nabla \times v^{T}\right), \\
g^{4}= & \nabla \cdot\left(v u^{T}\right)-\nabla \cdot\left(u v^{T}\right) .
\end{aligned}
$$

By the classical result in [10], we know that the essential spectrum of the operator $\mathcal{N}+G$ is relatively compact perturbation of $\mathcal{N}$ which has the essential spectrum

$$
\left\{\lambda \in \mathcal{C}^{2}: \lambda=\left(-|y|^{2}+i c y_{1},-|y|^{2}+i c y_{1}\right), y \in \mathbb{R}^{3}\right\} .
$$

Moreover, the spectra of $\mathcal{N}+G$ and $\mathcal{N}$ only differ by isolated eigenvalues of finite multiplicity. The above spectrum properties are critical to prove our main result. For convenience, we can rewrite (2.1) as

$$
\xi_{1} \omega_{t}=M_{1} \omega+g^{3}(\omega, u, v), \quad \xi_{2} v_{t}=M_{2} v+g^{4}(\omega, u, v),
$$

where $g^{3}$ and $g^{4}$ defined in (2.2)-(2.3),

$$
M_{1}=\overline{M_{1}}+g^{1}=\nu \triangle+c_{1} \partial_{x_{1}}+g^{1}, \quad M_{2}=\overline{M_{2}}+g^{2}=\eta \triangle+c_{1} \partial_{x_{1}}+g^{2} .
$$

We make the ansatz

$$
\omega(x, t)=\sum_{n \in \mathbf{Z}} \omega_{n}(x) e^{i n t}, \quad v(x, t)=\sum_{n \in \mathbf{Z}} v_{n}(x) e^{i n t}
$$

to (2.4), we obtain

$$
\left(i n \xi_{1}-M_{1}\right) \omega_{n}=g_{n}^{3}(\omega, u, v), \quad\left(i n \xi_{2}-M_{2}\right) v_{n}=g_{n}^{4}(\omega, u, v),
$$

where

$$
g^{3}(\omega, u, v)(x, t)=\sum_{n \in \mathbf{Z}} g_{n}^{3}(\omega, u, v) e^{\mathrm{int}}, \quad g^{4}(\omega, u, v)(x, t)=\sum_{n \in \mathbf{Z}} g_{n}^{4}(\omega, u, v) e^{\mathrm{int}}
$$


Note that we are interested in real valued solution only. We will always suppose that $\left(\omega_{n}, v_{n}\right)=\left(\omega_{-n}, v_{-n}\right)$ for $n \in \mathbf{Z}$. These series are uniformly convergent on $\mathbb{R}^{3} \times[0,2 \pi]$ in the spaces which we have chosen. More precisely, we have the following result:

Lemma 2.1. A linear operator $\boldsymbol{J}: \mathcal{X} \rightarrow \boldsymbol{C}_{b}^{0}\left(\mathbb{R}^{3} \times[0, \pi]\right)$ is defined by

$$
(\boldsymbol{J} u)(x, t)=\widetilde{u}(x, t):=\sum_{n \in \boldsymbol{Z}} u_{n}(x) e^{i n t}, \quad u=\left(u_{n}\right)_{n \in \boldsymbol{Z}} \in \mathcal{X} .
$$

Then $\boldsymbol{J}$ is bounded.

The counterpart of multiplication $u v$ in the physical space is given by the convolution $\left(\sum_{k \in \mathbf{Z}} u_{n-k} v_{k}\right)_{n \in \mathbf{Z}}$, since

$$
u v=\sum_{l \in \mathbf{Z}} u_{l}(x) e^{i l t} \sum_{j \in \mathbf{Z}} v_{j}(x) e^{i j t}=\sum_{n \in \mathbf{Z}}\left(\sum_{k \in \mathbf{Z}} u_{n-k}(x) v_{k}(x)\right) e^{i n t} .
$$

Lemma 2.2. For $u=\left(u_{n}\right)_{n \in \boldsymbol{Z}}, v=\left(v_{n}\right)_{n \in \boldsymbol{Z}} \in \boldsymbol{X}$, the convolution $u * v \in \boldsymbol{X}$ is defined by

$$
(u * v)_{n}=\sum_{k \in Z} u_{n-k} v_{k}, \quad n \in Z
$$

Then there exists $C>0$ such that

$$
\|u * v\|_{\mathcal{X}} \leq C\|u\|_{\mathcal{X}}\|v\|_{\mathcal{X}}
$$

Lemma 2.3. Let a linear operator $M_{i}: \boldsymbol{X} \rightarrow \boldsymbol{X}$ be defined component-wise as $\left(M_{i} u\right)_{n}=M_{i n} u_{n}$ for $u=\left(u_{n}\right)_{n \in Z}$. Then

$$
\left\|M_{i} u\right\|_{\boldsymbol{X}}=\left(\left\|M_{i 0}\right\|_{\boldsymbol{H}^{m} \rightarrow \boldsymbol{H}^{m}}+\sup _{n \in \boldsymbol{Z} \backslash\{0\}}\left\|M_{i}\right\|_{\boldsymbol{H}^{m} \rightarrow \boldsymbol{H}^{m}}\right)\|u\|_{\boldsymbol{X}}, \quad \text { for } i=1,2 .
$$

The proofs of above three lemmas are rather standard, so we omit it.

For any bounded analytic semigroup $A_{\gamma}^{\alpha}$, the following result holds.

Lemma 2.4 ([18]). For every $0<\gamma<1$ and $p>1$ there exists a constant $M>0$ such that for all $t>0$ one has

$$
\left\|A_{\sigma}^{\gamma} e^{A_{\sigma} t}\right\|_{L^{p} \rightarrow L^{p}} \leq \frac{M}{t^{\gamma}}
$$

The proof of following result can be found in [8] for bounded domain and [18] for $\mathbf{R}^{n}$.

Lemma 2.5. For every $1 / 2<\gamma<1$ and $p>1$ there exists a constant $C>0$ such that

$$
\left\|A_{\sigma}^{-\gamma} f\right\|_{L^{p}} \leq C\|f\|_{W^{-2 \gamma, p}}
$$

The following result shows a weighted Young theorem. 
LEMma 2.6. There exists a positive constant $C$ such that

$$
\|\widehat{\omega} * \widehat{u}\|_{\boldsymbol{L}_{m}^{2}} \leq C\|\widehat{\omega}\|_{\boldsymbol{L}_{m}^{2}}\|\widehat{u}\|_{\boldsymbol{L}_{m}^{2}}, \quad \text { i.e. } \quad\|\boldsymbol{\omega} u\|_{\boldsymbol{H}^{m}} \leq C\|\omega\|_{\boldsymbol{H}^{m}}\|u\|_{\boldsymbol{H}^{m}} .
$$

Proof. It is easy to check that

$$
\rho(x) \leq \rho(x-y) \rho(y), \quad x, y \in \mathbb{R}^{3},
$$

where we take the weighted function as $\rho(x)=\left(1+|x|^{2}\right)^{1 / 2}$. Then, there exist positive constants $s_{1}, s_{2}, s$ such that $s_{1}+s_{2}=m+s$, with $s_{1}, s_{2}, s<m$. Using Young inequality and (2.6), we have

$$
\begin{aligned}
\|\widehat{\omega} * \widehat{u}\|_{\mathbf{L}_{m}^{2}}^{2} & =\int_{\mathbb{R}^{3}} \rho^{2 m}(\widehat{\omega} * \widehat{u})^{2}(x) d x \\
& =\int_{\mathbb{R}^{3}}\left(\int_{\mathbb{R}^{3}} \widehat{\omega}(x-y) \widehat{u}(y) \rho^{2 m}(x) d y\right)^{2} d x \\
& =\int_{\mathbb{R}^{3}} \rho^{-2 s}(x)\left(\int_{\mathbb{R}^{3}} \rho^{s_{1}}(x-y) \widehat{\omega}(x-y) \rho^{s_{2}}(y) \widehat{u}(y) d y\right)^{2} d x \\
& \leq \int_{\mathbb{R}^{3}} \rho^{-2 s}(x)\left(\int_{\mathbb{R}^{3}} \rho^{2 s_{1}}(z) \widehat{\omega}^{2}(z) d z\right)\left(\int_{\mathbb{R}^{3}} \rho^{2 s_{2}}(y) \widehat{u}^{2}(y) d y\right) d x \\
& \leq C\left\|\widehat{\omega}_{\mathbf{L}_{s_{1}}^{2}}^{2}\right\| \widehat{u}\left\|_{\mathbf{L}_{s_{2}}^{2}}^{2} \leq C \widehat{\omega}^{2}\right\|_{\mathbf{L}_{m}^{2}}^{2}\|\widehat{u}\|_{\mathbf{L}_{m}^{2}}^{2} .
\end{aligned}
$$

This completes the proof.

\section{Proof of Theorem 1.1}

In this section, we will give the detail of proof of Theorem 1.1. By (H2) and (H3), we know that the operator $M_{i}$ has two eigenvalues $\lambda_{0}^{ \pm}(\beta)$ and all other eigenvalues of $M_{i}$ are strictly bounded away from the imaginary axis in the left half plane. Thus we construct a $M_{i}$-invariant projections $\mathbf{P}_{ \pm 1, c}$ by

$$
\begin{aligned}
P_{1, c} \omega & =\left(\psi^{+, *}, \omega\right)_{\mathbf{L}^{2}} \psi^{+}, & P_{-1, c} \omega & =\left(\psi^{-, *}, \omega\right)_{\mathbf{L}^{2}} \psi^{-}, \\
P_{1, c} v & =\left(\psi^{+, *}, v\right)_{\mathbf{L}^{2}} \psi^{+}, & P_{-1, c} v & =\left(\psi^{-, *}, v\right)_{\mathbf{L}^{2}} \psi^{-},
\end{aligned}
$$

where $\psi^{ \pm}$denotes the associated normalized eigenfunctions, $\psi^{ \pm 1, *}$ denotes the associated normalized eigenfunctions of the adjoint operator $M_{i}^{*}$. The bounded "stable" part of the projection is $\mathbf{P}_{ \pm 1, s}=I-\mathbf{P}_{ \pm 1, c}$, we also know that $\mathbf{P}_{ \pm, c} M_{i}=$ $M_{i} \mathbf{P}_{ \pm, c}$ and $\mathbf{P}_{ \pm, s} M_{i}=M_{i} \mathbf{P}_{ \pm, s}$. Thus we can split $\omega_{ \pm 1}$ and $v_{ \pm 1}$ as

$$
\begin{array}{ll}
\omega_{1}=\omega_{1, c}+\omega_{1, s}, & \omega_{-1}=\omega_{-1, c}+\omega_{-1, s}, \\
v_{1}=v_{1, c}+v_{1, s}, & v_{-1}=v_{-1, c}+v_{-1, s}
\end{array}
$$

with $\omega_{ \pm 1, c}=\mathbf{P}_{ \pm 1, c} \omega_{1}, \omega_{ \pm 1, s}=\mathbf{P}_{ \pm 1, s} \omega_{1}, v_{ \pm 1, c}=\mathbf{P}_{ \pm 1, c} v_{1}, v_{ \pm 1, s}=\mathbf{P}_{ \pm 1, s} v_{1}$.

Applying above decompositions to (3.35), we have

$$
\begin{aligned}
\left(i n \xi_{1}-M_{1}\right) \omega_{n} & =g_{n}^{3}(\omega, u, v), & & n= \pm 2, \pm 3, \ldots, \\
\left(i n \xi_{2}-M_{2}\right) v_{n} & =g_{n}^{4}(\omega, u, v), & & n= \pm 2, \pm 3, \ldots,
\end{aligned}
$$




$$
\begin{array}{rlrl}
M_{1} \omega_{0} & =g_{0}^{3}(\omega, u, v), & & n=0, \\
M_{2} v_{0} & =g_{0}^{4}(\omega, u, v), & n=0, \\
\left( \pm i \xi_{1}-M_{1}\right) \omega_{ \pm 1, s} & =\mathbf{P}_{ \pm 1, s} g_{ \pm 1}^{3}(\omega, u, v), & \\
\left( \pm i \xi_{2}-M_{2}\right) v_{ \pm 1, s} & =\mathbf{P}_{ \pm 1, s} g_{ \pm 1}^{4}(\omega, u, v), & \\
\left( \pm i \xi_{1}-M_{1}\right) \omega_{ \pm 1, c} & =\mathbf{P}_{ \pm 1, c} g_{ \pm 1}^{3}(\omega, u, v), & \\
\left( \pm i \xi_{2}-M_{2}\right) v_{ \pm 1, c} & =\mathbf{P}_{ \pm 1, c} g_{ \pm 1}^{4}(\omega, u, v) . &
\end{array}
$$

The organization of proof of Theorem 1.1 is that we first solve the equations (3.5)-(3.6). Then using the fixed point theorem to solve equations (3.3)-(3.4) and (3.7)-(3.8) which is nontrivial due to the nonlinear term $g_{n}^{3}(\omega, u, v)$ and $g_{n}^{4}(\omega, u, v)$. At last, we employ the implicit function theorem to solve equation (3.9)-(3.10). The process of solving equation (3.9)-(3.10) is inspired by the classical Hopf-bifurcation result [16].

Rewrite (3.3)-(3.10) as

$$
\begin{aligned}
(i n \Xi+\mathcal{N}+G) \varphi_{n} & =F_{n}(\varphi, u), & & n= \pm 2, \pm 3, \ldots, \\
(\mathcal{N}+G) \varphi_{0} & =F_{0}(\varphi, u), & & n=0, \\
( \pm i \Xi+\mathcal{N}+G) \varphi_{ \pm 1, s} & =\mathbf{P}_{ \pm 1, s} F_{ \pm 1}(\varphi, u), & & \\
( \pm i \Xi+\mathcal{N}+G) \varphi_{ \pm 1, c} & =\mathbf{P}_{ \pm 1, c} F_{ \pm 1}(\varphi, u) . & &
\end{aligned}
$$

Now we first solve the equation (3.12). The linear operator $\mathcal{N}$ has essential spectrum up to the imaginary axis, it can be be inverted in the following sense.

Lemma 3.1. For $j=1,2$ and $f=\left(f^{1}, f^{2}\right)^{T} \in\left(\boldsymbol{H}^{m-1} \times \boldsymbol{H}^{m-1}\right) \cap\left(\boldsymbol{L}^{1} \times \boldsymbol{L}^{1}\right)$, the equation

$$
\mathcal{N} \varphi=\partial_{j} f
$$

has a unique solution $\varphi=\mathcal{N}^{-1} \partial_{j} f \in \boldsymbol{H}^{m} \times \boldsymbol{H}^{m}$. Moreover,

$$
\|\varphi\|_{\boldsymbol{H}^{m} \times \boldsymbol{H}^{m}} \leq C\|f\|_{\left(\boldsymbol{H}^{m-1} \times \boldsymbol{H}^{m-1}\right) \cap\left(\boldsymbol{L}^{1} \times \boldsymbol{L}^{1}\right)} .
$$

Proof. Define a smooth cut-off function $\chi$ taking its value in $[0,1]$ as

$$
\chi(y):= \begin{cases}1 & \text { if }|y| \leq 1 \\ 0 & \text { if }|y| \geq 2\end{cases}
$$

We denote

$$
\left(\widehat{f}_{1}^{1}, \widehat{f}_{1}^{2}\right)=\left(\widehat{f}^{1} \chi, \widehat{f}^{2} \chi\right) \quad \text { and } \quad\left(\widehat{f}_{2}^{1}, \hat{f}_{2}^{2}\right)=\left(\widehat{f}^{1}(1-\chi), \widehat{f}^{2}(1-\chi)\right)
$$

with $\widehat{f}=\left(f^{1}, f^{2}\right)=\left(\widehat{f}_{1}^{1}+\widehat{f}_{2}^{1}, \widehat{f}_{1}^{2}+\widehat{f}_{2}^{2}\right)$. Then

$$
\begin{aligned}
& \widehat{\omega}_{1}(y)=\frac{i y_{j} \widehat{f}_{1}^{1}}{i n \xi_{1}-\nu|y|^{2}-i c_{1} y_{1}} \quad \text { and } \quad \widehat{\omega}_{2}(y)=\frac{i y_{j} \widehat{f}_{2}^{1}}{i n \xi_{1}-\nu|y|^{2}-i c_{1} y_{1}}, \\
& \widehat{v}_{1}(y)=\frac{i y_{j} \widehat{f}_{1}^{2}}{i n \xi_{2}-\eta|y|^{2}-i c_{1} y_{1}} \quad \text { and } \quad \widehat{v}_{2}(y)=\frac{i y_{j} \widehat{f}_{2}^{2}}{i n \xi_{2}-\eta|y|^{2}-i c_{1} y_{1}} .
\end{aligned}
$$


Then $(\omega, v)=\left(\omega_{1}+\omega_{2}, v_{1}+v_{2}\right)$. Moreover, it has

$$
\begin{aligned}
\left\|\omega_{1}\right\|_{\mathbf{H}^{m}}^{2} & =\left\|\widehat{\omega}_{1}\right\|_{\mathbf{L}_{m}^{2}}^{2}=\int_{\mathbf{R}^{2}} \frac{\left|y_{j}\right|^{2}|\widehat{f} \chi(y)|^{2}}{\left.\left|i n \xi_{1}-\nu\right| y\right|^{2}-\left.i c_{1} y_{1}\right|^{2}} \rho^{2 m}(y) d y \\
& \leq C\|f\|_{\mathbf{L}^{1}}^{2} \int_{|y| \leq 2} \frac{\left|y_{j}\right|^{2}}{r^{4}+c^{2} y_{1}^{2}} d y \leq C\|f\|_{\mathbf{L}^{1}}^{2},
\end{aligned}
$$

and

$$
\begin{aligned}
\left\|\omega_{2}\right\|_{\mathbf{H}^{m}}^{2} & =\left\|\widehat{\omega}_{2}\right\|_{\mathbf{L}_{m}^{2}}^{2}=\int_{\mathbf{R}^{2}} \frac{\left|y_{j}\right|^{2}|\widehat{f}(1-\chi(y))|^{2}}{\left.\left|i n \xi_{1}-\nu\right| y\right|^{2}-\left.i c_{1} y_{1}\right|^{2}} \rho^{2 m}(y) d y \\
& \leq C \int_{\mathbf{R}^{2}}|\widehat{f}(y)|^{2} \rho^{(2 m-1)}(y) d y \leq C\|f\|_{\mathbf{H}^{m-1}}^{2} .
\end{aligned}
$$

By the same process, we also can obtain

$$
\left\|v_{1}\right\|_{\mathbf{H}^{m}}^{2}=\left\|\widehat{v}_{1}\right\|_{\mathbf{L}_{m}^{2}}^{2} \leq C\|f\|_{\mathbf{L}^{1}}^{2}, \quad\left\|v_{2}\right\|_{\mathbf{H}^{m}}^{2}=\left\|\widehat{v}_{2}\right\|_{\mathbf{L}_{m}^{2}}^{2} \leq C\|f\|_{\mathbf{H}^{m-1}}^{2} .
$$

This completes the proof.

This lemma tells us that $\widehat{\mathcal{N}}\left(i y_{i}, i y_{i}\right)^{T}$. is bounded compact operator in from $\mathbf{L}_{m}^{2} \times \mathbf{L}_{m}^{2}$ to itself. Furthermore, the spectra of $\widehat{\mathcal{N}+G}$ and $\widehat{\mathcal{N}}$ only differ by isolated eigenvalues of finite multiplicity (see the book of Henry [10, p. 136]).

The following lemma gives the solvable of the equation (3.12).

Lemma 3.2. Assume that (H1)-(H3) holds. Then the equation (3.12) has a unique solution

$$
\varphi_{0}=(\mathcal{N}+G)^{-1} F_{0}(\varphi, u) .
$$

Moreover, $\left\|\varphi_{0}\right\|_{\boldsymbol{H}^{m} \times \boldsymbol{H}^{m}} \leq C\left\|y_{j}^{-1} I_{2 \times 2} \widehat{F_{0}(\varphi, u)}\right\|_{L_{m}^{2} \times \boldsymbol{L}_{m}^{2}}$.

Proof. Since the operator $\widehat{\mathcal{N}}^{-1} \widehat{G}: \mathbf{L}_{m}^{2} \times \mathbf{L}_{m}^{2} \rightarrow \mathbf{L}_{m}^{2} \times \mathbf{L}_{m}^{2}$ is compact, the operator $I+\widehat{\mathcal{N}}^{-1} \widehat{G}$ is Fredholm with index 0. If $\left(I+\widehat{\mathcal{N}}^{-1} \widehat{G}\right) \widehat{\varphi}=0$ had a nontrivial solution, then $(\widehat{\mathcal{N}}+\widehat{G}) \widehat{\varphi}=\widehat{\mathcal{N}}\left(I+\widehat{\mathcal{N}}^{-1} \widehat{G}\right) \widehat{\varphi}=0$ would also have a nontrivial solution. This would contradict (H1). Hence the Fredholm property implies that the existence of $\left(I+\widehat{\mathcal{N}}^{-1} \widehat{G}\right)^{-1}: \mathbf{L}_{m}^{2} \times \mathbf{L}_{m}^{2} \rightarrow \mathbf{L}_{m}^{2} \times \mathbf{L}_{m}^{2}$. Then we have

$$
\widehat{\mathcal{N}}\left(I+\widehat{\mathcal{N}}^{-1} \widehat{G}\right) \widehat{\varphi}=i y_{j} I_{2 \times 2} \widehat{f}
$$

where $I_{2 \times 2}$ is the unit matrix.

Thus, by Lemma 2.4, we obtain

$$
\begin{aligned}
& \|\varphi\|_{\mathbf{H}^{m} \times \mathbf{H}^{m}}=\|\widehat{\varphi}\|_{\mathbf{L}_{m}^{2} \times \mathbf{L}_{m}^{2}} \\
& \leq\left\|\left(I+\widehat{\mathcal{N}}^{-1} \widehat{G}\right)^{-1}\right\|_{\mathbf{L}_{m}^{2} \times \mathbf{L}_{m}^{2} \rightarrow \mathbf{L}_{m}^{2} \times \mathbf{L}_{m}^{2}}\left\|\widehat{\mathcal{N}}^{-1} i y_{j} I_{2 \times 2} \widehat{f}\right\|_{\mathbf{L}_{m}^{2} \times \mathbf{L}_{m}^{2}} \leq C\|\widehat{f}\|_{\mathbf{L}_{m}^{2} \times \mathbf{L}_{m}^{2}} .
\end{aligned}
$$

This completes the proof. 
The velocity field $u$ is defined in terms of the vorticity via the Biot-Savart law

$$
u(x)=-\frac{1}{4 \pi} \int_{\mathbb{R}^{3}} \frac{(x-y)^{\perp} \times \omega(y)}{|x-y|^{3}} d y, \quad x \in \mathbb{R}^{3} .
$$

LEMma 3.3. There exist a constant $C>0$ such that

$$
\|u\|_{\boldsymbol{H}^{m}} \leq C\|\omega\|_{\boldsymbol{H}^{m}}, \quad\left\|\partial_{x_{i}} u\right\|_{\boldsymbol{H}^{m}} \leq C\|\omega\|_{\boldsymbol{H}^{m}} .
$$

Proof. The related equation of the velocity $u$ and the vorticity $\omega$ is

$$
\nabla \times u=\omega, \quad \nabla \cdot u=0, \quad \nabla \cdot \omega=0 .
$$

This leads in Fourier space to

$$
\left(\begin{array}{ccc}
0 & -i y_{3} & i y_{2} \\
i y_{3} & 0 & -i y_{1} \\
-i y_{2} & i y_{1} & 0 \\
i y_{1} & i y_{2} & i y_{3}
\end{array}\right)\left(\begin{array}{c}
\widehat{u}_{1} \\
\widehat{u}_{2} \\
\widehat{u}_{3}
\end{array}\right)=\left(\begin{array}{c}
\widehat{\omega}_{1} \\
\widehat{\omega}_{2} \\
\widehat{\omega}_{3} \\
0
\end{array}\right)
$$

We can get

$$
\widehat{N} \widehat{\omega}=-\frac{1}{|y|^{2}}\left(\begin{array}{cccc}
0 & i y_{3} & -i y_{2} & i y_{1} \\
-i y_{3} & 0 & i y_{1} & i y_{2} \\
i y_{2} & -i y_{1} & 0 & i y_{3}
\end{array}\right)\left(\begin{array}{c}
\widehat{\omega}_{1} \\
\widehat{\omega}_{2} \\
\widehat{\omega}_{3}
\end{array}\right)=\left(\begin{array}{c}
\widehat{u}_{1} \\
\widehat{u}_{2} \\
\widehat{u}_{3}
\end{array}\right)=\widehat{u} .
$$

Using Hölder's inequality, for $1 / p_{1}+1 / p_{2}=1, p_{1}, p_{2}>1, s_{1}+s_{2}=2 m$ and $s_{1}, s_{2}>0$, we have

$$
\begin{aligned}
\|u\|_{\mathbf{H}^{m}}^{2} & =\|\widehat{u}\|_{\mathbf{L}_{m}^{2}}^{2} \leq C\left(\left\|\chi_{|y| \leq 1} \widehat{N}\right\|_{\mathbf{L}_{s_{1} / 2}^{2 p_{1}}}^{2}\|\widehat{\omega}\|_{\mathbf{L}_{s_{2} / 2}^{2 p_{2}}}^{2}+\left\|\chi_{|y| \geq 1} \widehat{N}\right\|_{\mathbf{L}^{\infty}}^{2}\|\widehat{\omega}\|_{\mathbf{L}_{m}^{2}}^{2}\right) \\
& \leq C\left(\left\|\chi_{|y| \leq 1} \widehat{N}\right\|_{\mathbf{L}_{s_{1} / 2}^{2 p_{1}}}^{2}+\left\|\chi_{|y| \geq 1} \widehat{N}\right\|_{\mathbf{L}^{\infty}}^{2}\right)\|\widehat{\omega}\|_{\mathbf{L}_{m}^{2}}^{2} \leq C\|\widehat{\omega}\|_{\mathbf{L}_{m}^{2}}^{2}=C\|\omega\|_{\mathbf{H}^{m}}^{2},
\end{aligned}
$$

where we use the weighted function $\rho(y)=|y|(1+|y|)^{1 / 2}$, the boundedness of $\left\|\chi_{|y| \geq 1} i y_{i} /|y|^{2}\right\|_{\mathbf{L}^{\infty}}^{2}$ and

$$
\begin{aligned}
\left\|\chi_{|y| \leq 1} \frac{i y_{i}}{|y|^{2}}\right\|_{\mathbf{L}_{s_{1} / 2}^{2 p_{1}}}^{2} & =\int_{|y| \leq 1}\left|\frac{i y_{i}}{|y|^{2}}\right|^{2 p_{1}} \rho^{p_{1} s} d y \\
& =\int_{|y| \leq 1}\left|\frac{i y_{i}}{|y|^{2}}\right|^{2 p_{1}}|y|^{p_{1} s}(1+|y|)^{p_{1} s / 2} d y \\
& \leq C \int_{0}^{1} \frac{\varrho^{2 p_{1}}}{\varrho^{4 p_{1}}} \varrho^{p_{1} s}(1+\varrho)^{p_{1} s} \varrho^{2} d \varrho \\
& =C \int_{0}^{1} \varrho^{p_{1} s-2 p_{1}+2}(1+\varrho)^{p_{1} s} \varrho^{2} d \varrho \leq \infty,
\end{aligned}
$$

for $p_{1} s-2 p_{1}+2>0$. The second estimate in (3.17) is followed by

$$
\left\|\partial_{x_{i}} u\right\|_{\mathbf{H}^{m}}=\left\|i y_{i} \widehat{u} \rho^{m}\right\|_{\mathbf{L}^{2}} \leq\left\|i y_{i} \widehat{N}\right\|_{\mathbf{L}^{\infty}}\|\widehat{\omega}\|_{\mathbf{L}_{m}^{2}} \leq C\|\omega\|_{\mathbf{H}^{m}} .
$$


From the form of the nonlinear terms $g^{3}$ and $g^{4}$, it is critical to estimate the term as $u v$ and $u^{2}$. For convenience, we derive some estimates about the nonlinear term $N^{1}(\varphi)=\varphi^{2}$ and $N^{2}(\varphi, \psi)=\varphi \psi$. This proof is similar with Lemma 4 in [3], so we omit it.

Lemma 3.4. Define $N^{1}: \mathcal{X} \rightarrow \mathcal{X}$ by $N^{1}\left(\varphi_{n}\right)=N^{1}\left(\boldsymbol{J} \varphi_{n}\right)$ and $N^{2}: \mathcal{X} \times \mathcal{X} \rightarrow \mathcal{X}$ by $N^{2}\left(\varphi_{n}\right)=N^{2}\left(\boldsymbol{J} \varphi_{n}, \boldsymbol{J} \psi_{n}\right)$ for $\varphi, \psi \in \mathcal{X}$. Then there exists $C>0$ such that

$$
\left\|N^{1}(\varphi)\right\|_{\mathcal{X}} \leq C\|\varphi\|_{\mathcal{X}}^{2}, \quad\left\|N^{2}(\varphi, \psi)\right\|_{\mathcal{X}} \leq C\|\psi\|_{\mathcal{X}}\left\|_{\varphi}\right\|_{\mathcal{X}}
$$

for $\varphi, \psi \in \mathcal{X}$ with $\|\varphi\|_{\mathcal{X}} \leq 1$ and $\|\psi\|_{\mathcal{X}} \leq 1$. Moreover, there exists $C>0$ such that

$$
\begin{aligned}
\left\|N^{1}\left(\varphi^{1}\right)-N^{1}\left(\varphi^{2}\right)\right\|_{\mathcal{X}} \leq & C\left(\left\|\varphi^{1}\right\|_{\mathcal{X}}+\left\|\varphi^{2}\right\|_{\mathcal{X}}\right)\left\|\varphi^{1}-\varphi^{2}\right\|_{\mathcal{X}} \\
\left\|N^{2}\left(\varphi^{1}, \psi^{1}\right)-N^{2}\left(\varphi^{2}, \psi^{2}\right)\right\|_{\mathcal{X}} \leq & C\left(\left\|\varphi^{1}\right\|_{\mathcal{X}}+\left\|\varphi^{2}\right\|_{\mathcal{X}}+\left\|\psi^{1}\right\|_{\mathcal{X}}+\left\|\psi^{2}\right\|_{\mathcal{X}}\right) \\
& \times\left(\left\|\varphi^{1}-\varphi^{2}\right\| \mathcal{X}+\left\|\psi^{1}-\psi^{2}\right\|_{\mathcal{X}}\right)
\end{aligned}
$$

for $\varphi^{1}, \varphi^{2}, \psi^{1}, \psi^{2} \in \mathcal{X}$ with $\left\|\varphi^{1}\right\|_{\mathcal{X}},\left\|\varphi^{2}\right\|_{\mathcal{X}},\left\|\psi^{1}\right\|_{\mathcal{X}},\left\|\psi^{2}\right\|_{\mathcal{X}} \leq 1$

Then we have the following result.

Lemma 3.5. Assume that $\xi$ close enough to $\xi_{0}$. Then there exists a constant $C>0$ such that, for $n \neq 0$,

$$
\begin{array}{r}
\left\|(i n \Xi+\mathcal{N})^{-1}\right\|_{\mathcal{X} \rightarrow \mathcal{X}} \leq C, \\
\left\|(i n \Xi+\mathcal{N}-G)^{-1}\right\|_{\mathcal{X} \rightarrow \mathcal{X}} \leq C, \\
\left\|(i n \Xi+\mathcal{N}-G)^{-1} \boldsymbol{P}_{ \pm 1, s}\right\|_{\mathcal{X} \rightarrow \mathcal{X}} \leq C .
\end{array}
$$

ProOF. We observe that the solution $\varphi$ of the equation $($ in $\Xi+\mathcal{N}) \varphi=f$ is given by

$$
\widehat{\varphi}(y)=\left(\begin{array}{cc}
i n \xi_{1}+\nu|y|^{2}-i c_{1} y_{1} & 0 \\
0 & i n \xi_{2}+\eta|y|^{2}-i c_{1} y_{1}
\end{array}\right)^{-1} \widehat{f}(y), \quad y \in \mathcal{R}^{3} .
$$

For $\delta=\min \left\{\nu^{2}, \eta^{2}\right\} \xi^{2} /\left(\xi^{2}+4 c_{1}^{2}\right)$, we have

$$
\begin{aligned}
\left.\left|i n \xi_{1}+\nu\right| y\right|^{2}-\left.i c_{1} y_{1}\right|^{2} & =\nu^{2}|y|^{4}+\left(c_{1} y_{1}+n \xi_{1}\right)^{2} \\
& \geq \frac{\omega^{2}}{4 c_{1}^{2}} \chi_{|y| \leq \omega /\left(2 c_{1}\right)}+\delta^{2}\left(1+|y|^{2}\right) \chi_{|y| \geq \omega /\left(2 c_{1}\right)}, \\
\left.\left|i n \xi_{2}+\eta\right| y\right|^{2}-\left.i c_{1} y_{1}\right|^{2} & =\eta^{2}|y|^{4}+\left(c_{1} y_{1}+n \xi_{2}\right)^{2} \\
& \geq \frac{\omega^{2}}{4 c_{1}^{2}} \chi_{|y| \leq \omega /\left(2 c_{1}\right)}+\delta^{2}\left(1+|y|^{2}\right) \chi_{|y| \geq \omega /\left(2 c_{1}\right)} .
\end{aligned}
$$

It follows for $f \in \mathbf{H}^{m} \times \mathbf{H}^{m}$ that $\widehat{\theta} \in \mathbf{L}_{m+2}^{2} \times \mathbf{L}_{m+2}^{2}$, thus $\theta \in \mathbf{H}^{m+2} \times \mathbf{H}^{m+2}$. 
Let $\widehat{f} \in \mathbf{L}_{m+2}^{2} \times \mathbf{L}_{m+2}^{2} \subset \mathbf{L}_{m}^{2} \times \mathbf{L}_{m}^{2}, \widehat{\bar{\omega}}=\rho(y, \varepsilon) \widehat{\omega}$ and $\widehat{\bar{v}}=\rho(y, \varepsilon) \widehat{v}$ with $\rho(x, \varepsilon)=\sqrt{1+\varepsilon|x|^{2}}$. Note that $\varphi$ is a solution of the equation $(\operatorname{in} \Xi+\mathcal{N}) \varphi=f$. By a direct computation, we have

$$
(i n \Xi+\widehat{\mathcal{N}} \widehat{\bar{\varphi}})+\varepsilon L(y, \varepsilon) \widehat{\bar{\varphi}}=\widehat{g},
$$

where $\bar{\varphi}=(\widehat{\omega}, \widehat{v})^{T}, \widehat{g}=\rho(y, \varepsilon) \widehat{f}$ and

$$
\begin{aligned}
& \varepsilon L(y, \varepsilon) \\
= & \left(\begin{array}{cc}
\left(i n \xi_{1}+\nu|y|^{2}-i c y_{1}\right)\left(1-\rho^{-1}(y, \varepsilon)\right) & 0 \\
0 & \left(i n \xi_{2}+\eta|y|^{2}-i c y_{1}\right)\left(1-\rho^{-1}(y, \varepsilon)\right)
\end{array}\right) .
\end{aligned}
$$

Here we use the fact that $\mathcal{N}$ is elliptic of order of 2 . Hence it derives from the form of $\rho(y, \varepsilon)=\sqrt{1+\varepsilon|y|^{2}}$ that

$$
L(y, \varepsilon) \sim \frac{\varepsilon|y|^{4}}{1+\varepsilon|y|^{2}+\sqrt{1+\varepsilon|y|^{2}}}\left(\begin{array}{cc}
\nu_{0} & 0 \\
0 & \kappa_{0}
\end{array}\right) .
$$

Using a Neumann series, it derives from the boundness of the operator $L: \mathbf{L}_{m+2}^{2} \times$ $\mathbf{L}_{m+2}^{2} \rightarrow \mathbf{L}_{m}^{2} \times \mathbf{L}_{m}^{2}$ that

$$
(i n \Xi+\widehat{\mathcal{N}})+\varepsilon L: \mathbf{L}_{m+2}^{2} \times \mathbf{L}_{m+2}^{2} \rightarrow \mathbf{L}_{m}^{2} \times \mathbf{L}_{m}^{2}
$$

is invertible with a bounded inverse, for sufficient small $\varepsilon>0$. This implies that $\bar{\varphi} \in \mathbf{L}_{m+2}^{2} \times \mathbf{L}_{m+2}^{2}$, i.e. $\varphi \in \mathbf{H}^{m+2} \times \mathbf{H}^{m+2}$. Moreover, we have

$$
\begin{aligned}
\|\varphi\|_{\mathbf{H}^{m+2} \times \mathbf{H}^{m+2}} & =\|\widehat{\varphi}\|_{\mathbf{L}_{m+2}^{2} \times \mathbf{L}_{m+2}^{2}}=\|\widehat{\varphi}\|_{\mathbf{L}_{m+2}^{2} \times \mathbf{L}_{m+2}^{2}} \\
& \leq C\|\widehat{g}\|_{\mathbf{L}_{m}^{2} \times \mathbf{L}_{m}^{2}}=C\|f\|_{\mathbf{H}^{m+2} \times \mathbf{H}^{m+2}} .
\end{aligned}
$$

Above result shows that $(i n \Xi+\mathcal{N})^{-1}: \mathbf{H}^{m} \times \mathbf{H}^{m} \rightarrow \mathbf{H}^{m+2} \times \mathbf{H}^{m+2}$ is bounded. But we only need this operator to be bounded $\mathcal{X} \rightarrow \mathcal{X}$. This implies that the spectrum of $\mathcal{N}$ in $\mathcal{X}$ well separated from $i n \Xi$ for $n \neq 0$ and $\varepsilon>0$ sufficient small. In a similar manner to prove the first inequality, the rest two inequalities can be obtained, so we omit it.

By the same proof in Lemma 3.3, we obtain the following result.

Lemma 3.6. Assume that $\xi_{i}$ close enough to $\xi_{0}$ for $i=1,2$. Then there exists a constant $C>0$ such that

$$
\begin{array}{rr}
\left\|\left(i n \xi_{i}-\overline{M_{i}}\right)^{-1}\right\|_{\boldsymbol{H}^{m} \rightarrow \boldsymbol{H}^{m}} \leq C, & \left\|\left(i n \xi_{i}-\overline{M_{i}}\right)^{-1} \nabla^{j} \cdot\right\|_{\boldsymbol{H}^{m} \rightarrow \boldsymbol{H}^{m}} \leq C, \\
\left\|\left(i n \xi_{i}-M_{i}\right)^{-1}\right\|_{\boldsymbol{H}^{m} \rightarrow \boldsymbol{H}^{m}} \leq C, & \left\|\left(i n \xi_{i}-M_{i}\right)^{-1} \nabla^{j} \cdot\right\|_{\boldsymbol{H}^{m} \rightarrow \boldsymbol{H}^{m}} \leq C, \\
\left\|\left(i n \xi_{i}-M_{i}\right)^{-1} \boldsymbol{P}_{ \pm 1, s}\right\|_{\boldsymbol{H}^{m} \rightarrow \boldsymbol{H}^{m}} \leq C, & \left\|\left(i n \xi_{i}-M_{i}\right)^{-1} \nabla^{j} \cdot \boldsymbol{P}_{ \pm 1, s}\right\|_{\boldsymbol{H}^{m} \rightarrow \boldsymbol{H}^{m}} \leq C,
\end{array}
$$

for $n \neq 0$ and $j=1,2$. 
Thus by Lemmas 2.4 and 2.5, we can obtain the solution of equations (3.11) and (3.13) as

$$
\begin{aligned}
\varphi_{n} & =(i n \Xi+\mathcal{N})^{-1} F_{n}(\varphi, u), \quad n= \pm 2, \pm 3, \ldots, \\
\varphi_{ \pm 1, s} & =( \pm i \Xi+\mathcal{N})^{-1} \mathbf{P}_{ \pm 1, s} F_{ \pm 1}(\varphi, u),
\end{aligned}
$$

i.e.

$$
\begin{aligned}
\omega_{n} & =\left(i n \xi_{1}-M_{1}\right)^{-1} g_{n}^{3}(\omega, u, v), & n= \pm 2, \pm 3, \ldots, \\
v_{n} & =\left(i n \xi_{2}-M_{2}\right)^{-1} g_{n}^{4}(\omega, u, v), & n= \pm 2, \pm 3, \ldots, \\
\omega_{ \pm 1, s} & =\left( \pm i \xi_{1}-M_{1}\right)^{-1} \mathbf{P}_{ \pm 1, s} g_{ \pm 1}^{3}(\omega, u, v), & \\
v_{ \pm 1, s} & =\left( \pm i \xi_{2}-M_{2}\right)^{-1} \mathbf{P}_{ \pm 1, s} g_{ \pm 1}^{4}(\omega, u, v) . &
\end{aligned}
$$

The following lemma shows the solvable of equations (3.22)-(3.24).

Lemma 3.7. Assume that there exist $\sigma_{1}, \sigma_{2}>0$ such that for all $\xi_{1}, \xi_{2}>0$ with $\left|\xi_{1}-\xi_{0}\right|,\left|\xi_{2}-\xi_{0}\right| \leq \sigma_{1}$ and all $\omega_{ \pm 1, c}, v_{ \pm 1, c} \in \boldsymbol{H}^{m}$ with $\left\|\omega_{ \pm 1, c}\right\|_{\boldsymbol{H}^{m}},\left\|v_{ \pm 1, c}\right\|_{\boldsymbol{H}^{m}}$ $\leq \sigma_{2}$. Then equations (3.21)-(3.24) has a unique solution $(\widetilde{\omega}, \widetilde{v})=\Phi\left(\omega_{c}, v_{c}\right) \in \mathcal{X}$, where

$$
\begin{array}{ll}
\omega_{c}=\left(\omega_{-1, c}, \omega_{1, c}\right), \quad \widetilde{\omega}=\left(\ldots, \omega_{-2}, \omega_{-1, c}+\omega_{-1, s}, \omega_{0}, \omega_{1, c}+\omega_{1, s}, \omega_{2}, \ldots\right), \\
v_{c}=\left(v_{-1, c}, v_{1, c}\right), \quad \widetilde{v}=\left(\ldots, v_{-2}, v_{-1, c}+v_{-1, s}, v_{0}, v_{1, c}+v_{1, s}, v_{2}, \ldots\right) .
\end{array}
$$

Moreover, there exits $C>0$ such that

$$
\begin{aligned}
\Phi(0,0) & =(0,0), \\
\left\|\widetilde{\omega}-\omega_{c}\right\|_{\boldsymbol{X}} & \leq C\left(\left\|\omega_{-1, c}\right\|_{\boldsymbol{H}^{m}}^{2}+\left\|\omega_{1, c}\right\|_{\boldsymbol{H}^{m}}^{2}\right), \\
\left\|\widetilde{v}-v_{c}\right\|_{\boldsymbol{X}} & \leq C\left(\left\|v_{-1, c}\right\|_{\boldsymbol{H}^{m}}^{2}+\left\|v_{1, c}\right\|_{\boldsymbol{H}^{m}}^{2}\right),
\end{aligned}
$$

and

$$
\begin{gathered}
\|\widetilde{\omega}\|_{\boldsymbol{X}} \leq C\left(\left\|\omega_{-1, c}\right\|_{\boldsymbol{H}^{m}}^{2}+\left\|\omega_{1, c}\right\|_{\boldsymbol{H}^{m}}^{2}\right), \\
\|\widetilde{u}\|_{\boldsymbol{X}} \leq C\left(\left\|\omega_{-1, c}\right\|_{\boldsymbol{H}^{m}}^{2}+\left\|\omega_{1, c}\right\|_{\boldsymbol{H}^{m}}^{2}\right), \\
\|\widetilde{v}\|_{\boldsymbol{X}} \leq C\left(\left\|v_{-1, c}\right\|_{\boldsymbol{H}^{m}}^{2}+\left\|v_{1, c}\right\|_{\boldsymbol{H}^{m}}^{2}\right),
\end{gathered}
$$

with $\widetilde{\omega}-\omega_{c}:=\left(\ldots, 0, \omega_{-1, c}, 0, \omega_{1, c}, 0, \ldots\right), \widetilde{v}-v_{c}:=\left(\ldots, 0, v_{-1, c}, 0, v_{1, c}, 0, \ldots\right)$.

Proof. For fixed $\xi_{1}, \xi_{2}>0$ so close to $\xi_{0}$ and given $\omega_{ \pm 1, c}, v_{ \pm 1, c} \in \mathbf{H}^{m}$ with $\left\|\omega_{ \pm 1, c}\right\|_{\mathbf{H}^{m}},\left\|v_{ \pm 1, c}\right\|_{\mathbf{H}^{m}} \leq \sigma_{2}$. Define the operator $\Gamma:\left(\widetilde{\omega}^{*}, \widetilde{v}^{*}\right) \mapsto(\widetilde{\omega}, \widetilde{v})$

$$
\begin{aligned}
& =\left(\widetilde{\omega}^{*}+\left(\ldots, 0, \omega_{-1, c}, 0, \omega_{1, c}, 0, \ldots\right), \widetilde{v}^{*}+\left(\ldots, 0, v_{-1, c}, 0, v_{1, c}, 0, \ldots\right)\right) \\
& \mapsto(\omega, v) \mapsto\left(\widetilde{\omega}^{* *}, \widetilde{v}^{* *}\right)=\text { right hand side of }(3.21)-(3.24),
\end{aligned}
$$

where $(\omega, v)=(\mathbf{J} \widetilde{\omega}, \mathbf{J} \widetilde{v})$ are defined in Lemma 2.1 and

$$
\left(\widetilde{\omega}^{*}, \widetilde{v}^{*}\right)=\left(\left(\ldots, \omega_{-2}, \omega_{-1, s}, \omega_{0}, \omega_{1, s}, \omega_{2}, \ldots\right),\left(\ldots, v_{-2}, v_{-1, s}, v_{0}, v_{1, s}, v_{2}, \ldots\right)\right),
$$




$$
\begin{aligned}
(\widetilde{\omega}, \widetilde{v}) & =\left(\widetilde{\omega}^{*}+\omega_{c}, \widetilde{v}^{*}+v_{c}\right) \\
& =\left(\widetilde{\omega}^{*}+\left(\ldots, 0, \omega_{-1, c}, 0, \omega_{1, c}, 0, \ldots\right), \widetilde{v}^{*}+\left(\ldots, 0, v_{-1, c}, 0, v_{1, c}, 0, \ldots\right)\right) .
\end{aligned}
$$

Now we prove the operator $\Gamma$ is a self-map of a sufficiently small ball in $\mathcal{X}$. Using Lemmas 2.6, 3.1, 3.2 and Lemma 3.4, by the form of nonlinear terms $g^{3}$ and $g^{4}$ in $(2.2)-(2.3)$, respectively, we derive

$$
\begin{aligned}
\left\|\widetilde{\omega}^{* *}\right\|_{\mathbf{X}} \leq & C \sup \left\{\left\|\left(i n \xi_{1}-M_{1}\right)^{-1}\right\|_{\mathbf{H}^{m} \rightarrow \mathbf{H}^{m}},\right. \\
& \left\|\left( \pm i \xi_{1}-M_{1}\right)^{-1} \mathbf{P}_{ \pm 1, s}\right\|_{\mathbf{H}^{m} \rightarrow \mathbf{H}^{m}}, \\
& \left\|\left(i n \xi_{1}-M_{1}\right)^{-1} \nabla^{j}\right\|_{\mathbf{H}^{m} \rightarrow \mathbf{H}^{m}}, \\
& \left.\left\|\left( \pm i \xi_{1}-M_{1}\right)^{-1} \nabla^{j} \mathbf{P}_{ \pm 1, s}\right\|_{\mathbf{H}^{m} \rightarrow \mathbf{H}^{m}}: n \in \mathbf{Z} \backslash\{-1,1\}\right\} \\
& \times\left\|\left(\widetilde{g}_{n}^{3}(\omega, u, v)\right)_{n \in \mathbf{Z}}\right\|_{\mathbf{X}} \\
\leq & C\left\|\widetilde{g}^{3}(\widetilde{\omega}, u, \widetilde{v})\right\|_{\mathbf{X}} \\
\leq & C\left(\|\widetilde{\omega}\|_{\mathbf{X}}\|u\|_{\mathbf{X}}+\|\widetilde{\omega}\|_{\mathbf{X}}\|\widetilde{v}\|_{\mathbf{X}}+\|u\|_{\mathbf{X}}\|\widetilde{v}\|_{\mathbf{X}}\right) \\
\leq & C\left(\|\widetilde{\omega}\|_{\mathbf{X}}^{2}+\|\widetilde{\omega}\|_{\mathbf{X}}\|\tilde{v}\|_{\mathbf{X}}\right) \\
\leq & C\left(\left\|\widetilde{\omega}^{*}\right\|_{\mathbf{X}}^{2}+\left\|\omega_{-1, c}\right\|_{\mathbf{H}^{m}}^{2}+\left\|\omega_{1, c}\right\|_{\mathbf{H}^{m}}^{2}\right. \\
& \left.+\left\|\widetilde{v}^{*}\right\|_{\mathbf{X}}^{2}+\left\|v_{-1, c}\right\|_{\mathbf{H}^{m}}^{2}+\left\|v_{1, c}\right\|_{\mathbf{H}^{m}}^{2}\right) \\
\leq & C\left(\left\|\widetilde{\omega}^{*}\right\|_{\mathbf{X}}^{2}+\left\|\widetilde{v}^{*}\right\|_{\mathbf{X}}^{2}+\sigma_{2}^{2}\right), \\
\left\|\widetilde{v}^{* *}\right\|_{\mathbf{X}} \leq & C \sup ^{2}\left\|\left(i n \xi_{2}-M_{2}\right)^{-1}\right\|_{\mathbf{H}^{m} \rightarrow \mathbf{H}^{m}}, \\
& \left\|\left( \pm i \xi_{2}-M_{2}\right)^{-1} \mathbf{P}_{ \pm 1, s}\right\|_{\mathbf{H}^{m} \rightarrow \mathbf{H}^{m}}, \\
& \left\|\left(i n \xi_{2}-M_{2}\right)^{-1} \nabla^{j}\right\|_{\mathbf{H}^{m} \rightarrow \mathbf{H}^{m}}, \\
& \left.\left\|\left( \pm i \xi_{2}-M_{2}\right)^{-1} \nabla^{j} \mathbf{P}_{ \pm 1, s}\right\|_{\mathbf{H}^{m} \rightarrow \mathbf{H}^{m}}: n \in \mathbf{Z} \backslash\{-1,1\}\right\} \\
& \times\left\|\left(\widetilde{g}_{n}^{4}(\omega, u, v)\right)_{n \in \mathbf{Z}}\right\|_{\mathbf{X}} \\
\leq & C\left\|\widetilde{g}^{4}(\widetilde{\omega}, u, \widetilde{v})\right\|_{\mathbf{X}} \leq C\left(\|\widetilde{v}\|_{\mathbf{X}}^{2}+\|\widetilde{\omega}\|_{\mathbf{X}}\|\widetilde{v}\|_{\mathbf{X}}\right) \\
\leq & C\left(\left\|\widetilde{\omega}^{*}\right\|_{\mathbf{X}}^{2}+\left\|\omega_{-1, c}\right\|_{\mathbf{H}^{m}}^{2}+\left\|\omega_{1, c}\right\|_{\mathbf{H}^{m}}^{2}\right. \\
& \left.+\left\|\widetilde{v}^{*}\right\|_{\mathbf{X}}^{2}+\left\|v_{-1, c}\right\|_{\mathbf{H}^{m}}^{2}+\left\|v_{1, c}\right\|_{\mathbf{H}^{m}}^{2}\right) \\
\leq & C\left(\left\|\widetilde{\omega}^{*}\right\|_{\mathbf{X}}^{2}+\left\|\widetilde{v}^{*}\right\|_{\mathbf{X}}^{2}+\sigma_{2}^{2}\right),
\end{aligned}
$$

where $\widetilde{g}^{3}=C\left(\omega u^{T}+u \omega^{T}+|v|^{2} / 2+v v^{T}\right), \widetilde{g}^{4}=C\left(v u^{T}-u v^{T}\right)$. Thus, for $\sigma_{2} \leq 1 / \sqrt{2 C}$ and $\left(\widetilde{\omega}^{*}, \widetilde{v}^{*}\right) \in \mathcal{X}$ with $\left\|\left(\widetilde{\omega}^{*}, \widetilde{v}^{*}\right)\right\|_{\mathcal{X}} \leq 1 / \sqrt{2 C}$, we have

$$
\left\|\Gamma\left(\widetilde{\omega}^{*}, \widetilde{v}^{*}\right)\right\|_{\mathcal{X}}=\left\|\widetilde{\omega}^{* *}\right\|_{\mathcal{X}}+\left\|\widetilde{v}^{* *}\right\|_{\mathbf{X}} \leq C\left(\left(\left\|\widetilde{\omega}^{*}\right\|_{\mathbf{X}}+\left\|\widetilde{v}^{*}\right\|_{\mathbf{X}}\right)^{2}+\sigma_{2}^{2}\right) \leq 1,
$$

which implies that for sufficient small $\sigma_{2}>0, \Gamma$ maps the $\|\cdot\|_{\mathcal{X}}$ ball of radius $r=1$. Hence, we obtain a unique fixed point $\left(\widetilde{\theta}^{*}, \widetilde{v}^{*}\right) \in \mathcal{X}$ of $\Gamma$, which means that equations (3.21)-(3.24) has solution of $(\widetilde{\omega}, \widetilde{v})=\left(\widetilde{\omega}^{*}+\omega_{c}, \widetilde{v}^{*}+v_{c}\right)$. Moreover, if $\left(\omega_{ \pm 1, c}, v_{ \pm 1, c}\right)=(0,0)$, then $\Phi(0,0)=(0,0)$. Next we prove the second inequality 
in (3.25). Note that

$$
\left(\widetilde{\omega}^{*}, \widetilde{v}^{*}\right)=\Gamma\left(\widetilde{\omega}^{*}, \widetilde{v}^{*}\right)=\left(\widetilde{\theta}^{* *}, \widetilde{v}^{* *}\right),
$$

which combine with (3.31)-(3.32), we derive

$$
\begin{aligned}
\left\|\widetilde{\omega}-\omega_{c}\right\|_{\mathbf{X}} & =\left\|\widetilde{\omega}^{*}\right\|_{\mathbf{X}}=\left\|\widetilde{\omega}^{* *}\right\|_{\mathbf{X}} \leq C\left(\left\|\widetilde{\omega}^{*}\right\|_{\mathbf{X}}^{2}+\left\|\omega_{-1, c}\right\|_{\mathcal{H}^{m}}^{2}+\left\|\omega_{1, c}\right\|_{\mathcal{H}^{m}}^{2}\right), \\
\left\|\widetilde{v}-v_{c}\right\|_{\mathbf{X}} & =\left\|\widetilde{v}^{*}\right\|_{\mathbf{X}}=\left\|\widetilde{v}^{* *}\right\|_{\mathbf{X}} \leq C\left(\left\|\widetilde{v}^{*}\right\|_{\mathbf{X}}^{2}+\left\|v_{-1, c}\right\|_{\mathcal{H}^{m}}^{2}+\left\|v_{-1, c}\right\|_{\mathcal{H}^{m}}^{2}\right) .
\end{aligned}
$$

Thus we deduce that for sufficient small ball $\mathbf{B}_{r}(0) \subset \mathbf{B}_{1}(0)$,

$$
\begin{aligned}
\left\|\widetilde{\omega}-\omega_{c}\right\|_{\mathbf{x}} & \leq C\left(\left\|\omega_{-1, c}\right\|_{\mathbf{H}^{m}}^{2}+\left\|\omega_{1, c}\right\|_{\mathbf{H}^{m}}^{2}\right), \\
\left\|\widetilde{v}-v_{c}\right\| \mathbf{x} & \leq C\left(\left\|v_{-1, c}\right\|_{\mathbf{H}^{m}}^{2}+\left\|v_{1, c}\right\|_{\mathbf{H}^{m}}^{2}\right) .
\end{aligned}
$$

Note that

$\widetilde{\omega}-\omega_{c}:=\left(\ldots,, 0, \omega_{-1, c}, 0, \omega_{1, c}, 0, \ldots\right), \quad \widetilde{v}-v_{c}:=\left(\ldots,, 0, v_{-1, c}, 0, v_{1, c}, 0, \ldots\right)$.

Hence by (3.33)-(3.34) and Lemma 2.6, we obtain

$$
\left\|\omega_{c}\right\|_{\mathbf{X}} \leq C\left(\left\|\omega_{-1, c}\right\|_{\mathbf{H}^{m}}^{2}+\left\|\omega_{1, c}\right\|_{\mathbf{H}^{m}}^{2}\right), \quad\left\|v_{c}\right\|_{\mathbf{X}} \leq C\left(\left\|v_{-1, c}\right\|_{\mathbf{H}^{m}}^{2}+\left\|v_{1, c}\right\|_{\mathbf{H}^{m}}^{2}\right),
$$

and

$$
\begin{aligned}
\|\widetilde{\omega}\|_{\mathbf{X}} & \leq C\left(\left\|\omega_{-1, c}\right\|_{\mathbf{H}^{m}}^{2}+\left\|\omega_{1, c}\right\|_{\mathbf{H}^{m}}^{2}\right), \\
\|\widetilde{u}\|_{\mathbf{X}} & \leq C\left(\left\|\omega_{-1, c}\right\|_{\mathbf{H}^{m}}^{2}+\left\|\omega_{1, c}\right\|_{\mathbf{H}^{m}}^{2}\right), \\
\|\widetilde{v}\|_{\mathbf{X}} & \leq C\left(\left\|v_{-1, c}\right\|_{\mathbf{H}^{m}}^{2}+\left\|v_{1, c}\right\|_{\mathbf{H}^{m}}^{2}\right) .
\end{aligned}
$$

This completes the proof.

In the following, we can complete our proof of Theorem 1.1.

Proof OF Theorem 1.1. To prove Theorem 1.1, the rest remains to analyze equations (3.9)-(3.10). We restate equations:

$$
\begin{aligned}
\left( \pm i \xi_{1}-M_{1}\right) \omega_{ \pm 1, c} & =\mathbf{P}_{ \pm 1, c} g_{ \pm 1}^{3}(\omega, u, v), \\
\left( \pm i \xi_{2}-M_{2}\right) v_{ \pm 1, c} & =\mathbf{P}_{ \pm 1, c} g_{ \pm 1}^{4}(\omega, u, v) .
\end{aligned}
$$

It follows from $\left(\omega_{-1}, v_{-1}\right)=\left(\overline{\omega_{1}}, \overline{v_{1}}\right)$ and $\left(g_{ \pm 1}^{3}, g_{ \pm 1}^{4}\right)=\left(\overline{g_{ \pm 1}^{3}}, \overline{g_{ \pm 1}^{4}}\right)$ that the "-" equation is the complex conjugate of the "+" equation. By Lemma 2.1, we can denote $(\omega, v)=(\mathbf{J} \widetilde{\omega}, \mathbf{J} \widetilde{v})$ by means of

$$
(\widetilde{\omega}, \widetilde{v})=\Phi\left(\omega_{c}, v_{c}\right)=\Phi\left(\left(\overline{\omega_{1, c}}, \omega_{1, c}\right),\left(\overline{v_{1, c}}, v_{1, c}\right)\right) .
$$

Our target is to find $\left(\xi_{1}, \beta\right)$ and $\left(\xi_{2}, \beta\right)$ close to $\left(\xi_{0}, \beta_{c}\right)$ and a nontrivial solution $\left(\omega_{1, c}, v_{1, c}\right)=\left(\omega_{1, c}, v_{1, c}\right)(x)$ of

$$
\begin{aligned}
-i \xi_{1} \omega_{1, c}+M_{1} \omega_{1, c}+\mathbf{P}_{1, c} g_{1}^{3}\left(\mathbf{J} \Phi\left(\overline{\omega_{1, c}}, \omega_{1, c}, \overline{v_{1, c}}, v_{1, c}\right)\right) & =0, \\
-i \xi_{2} v_{1, c}+M_{2} v_{1, c}+\mathbf{P}_{1, c} g_{1}^{4}\left(\mathbf{J} \Phi\left(\overline{\omega_{1, c}}, \omega_{1, c}, \overline{v_{1, c}}, v_{1, c}\right)\right) & =0 .
\end{aligned}
$$


Due to $\omega_{1, c}, v_{1, c} \in \mathrm{C} \psi^{+}$and $\left(M_{1} \psi^{+}, M_{2} \psi^{+}\right)=\left(\lambda_{0}^{+}(\beta) \psi^{+}, \mu_{0}^{+}(\beta) \psi^{+}\right)$, we can write

$$
\omega_{1, c}=\eta \psi^{+}, \quad v_{1, c}=\delta \psi^{+} .
$$

Then by (3.35)-(3.36), we obtain

$$
\begin{aligned}
& -i \xi_{1} \eta \psi^{+}+\lambda_{0}^{+}(\beta) \eta \psi^{+}+\mathbf{P}_{1, c} g_{1}^{3}\left(\mathbf{J} \Phi\left(\overline{\eta \psi^{+}}, \eta \psi^{+}, \overline{\delta \psi^{+}}, \delta \psi^{+}\right)\right)=0, \\
& -i \xi_{2} \delta \psi^{+}+\mu_{0}^{+}(\beta) \delta \psi^{+}+\mathbf{P}_{1, c} g_{1}^{4}\left(\mathbf{J} \Phi\left(\overline{\eta \psi^{+}}, \eta \psi^{+}, \overline{\delta \psi^{+}}, \delta \psi^{+}\right)\right)=0,
\end{aligned}
$$

for some $\eta, \delta \in \mathrm{C} \backslash\{0\}$.

To be simple, we introduce $\left(p_{1, c}, \theta_{1, c}\right)$ by

$$
\left(\mathbf{P}_{1, c} \omega, \mathbf{P}_{1, c} v\right)=\left(p_{1, c}(\omega) \psi^{+}, \theta_{1, c}(v) \psi^{+}\right) .
$$

Then equations (3.37)-(3.38) can be written as

$$
\begin{array}{ll}
-i \xi_{1} \eta+\lambda_{0}^{+}(\beta) \eta+g^{3}(\beta, \eta, \delta)=0, & \text { for some } \eta \in \mathrm{C}, \\
-i \xi_{2} \delta+\mu_{0}^{+}(\beta) \delta+g^{4}(\beta, \eta, \delta)=0, & \text { for some } \delta \in \mathrm{C},
\end{array}
$$

where the cubic coefficient $\mu \neq 0$ in

$$
\begin{aligned}
& g^{3}(\beta, \eta, \delta):=p_{1, c}\left(g_{1}^{3}\left(\mathbf{J} \Phi\left(\overline{\eta \psi^{+}}, \eta \psi^{+}, \overline{\delta \psi^{+}}, \delta \psi^{+}\right)\right),\right. \\
& g^{4}(\beta, \eta, \delta):=p_{1, c}\left(g_{1}^{4}\left(\mathbf{J} \Phi\left(\overline{\eta \psi^{+}}, \eta \psi^{+}, \overline{\delta \psi^{+}}, \delta \psi^{+}\right)\right) .\right.
\end{aligned}
$$

Note that

$$
\begin{aligned}
\left|p_{1, c}(\omega)\right| & \leq C\left\|\mathbf{P}_{1, c} \omega\right\|_{\mathbf{H}^{m}} \leq C\|\omega\|_{\mathbf{H}^{m}}, \\
\left|p_{1, c}(v)\right| & \leq C\left\|\mathbf{P}_{1, c} v\right\|_{\mathbf{H}^{m}} \leq C\|v\|_{\mathbf{H}^{m}} .
\end{aligned}
$$

So by (3.28)-(3.30), (3.43)-(3.44), we derive

$$
\begin{aligned}
& \mid p_{1, c}\left(g_{1}^{3}\left(\mathbf{J} \Phi\left(\overline{\eta \psi^{+}}, \eta \psi^{+}, \overline{\delta \psi^{+}}, \delta \psi^{+}\right)\right) \mid \leq C \| g_{1}^{3}\left(\mathbf{J} \Phi\left(\overline{\eta \psi^{+}}, \eta \psi^{+}, \overline{\delta \psi^{+}}, \delta \psi^{+}\right) \|_{\mathbf{H}^{m}}\right.\right. \\
& \leq C\left\|\Phi\left(\overline{\eta \psi^{+}}, \eta \psi^{+}, \overline{\delta \psi^{+}}, \delta \psi^{+}\right)\right\|_{\mathcal{X}} \\
& \leq C\left(\left\|\overline{\omega_{1, c}}\right\|_{\mathbf{H}^{m}}^{2}+\left\|\omega_{1, c}\right\|_{\mathbf{H}^{m}}^{2}+\left\|\overline{v_{1, c}}\right\|_{\mathbf{H}^{m}}^{2}+\left\|v_{1, c}\right\|_{\mathbf{H}^{m}}^{2}\right) \\
& \leq C\left(\left\|\eta \psi^{+}\right\|_{\mathbf{H}^{m}}^{2}+\left\|\delta \psi^{+}\right\|_{\mathbf{H}^{m}}^{2}\right) \leq C\left(|\eta|^{2}+|\delta|^{2}\right), \\
& \mid p_{1, c}\left(g_{1}^{4}\left(\mathbf{J} \Phi\left(\overline{\eta \psi^{+}}, \eta \psi^{+}, \overline{\delta \psi^{+}}, \delta \psi^{+}\right)\right) \mid \leq C \| g_{1}^{4}\left(\mathbf{J} \Phi\left(\overline{\eta \psi^{+}}, \eta \psi^{+}, \overline{\delta \psi^{+}}, \delta \psi^{+}\right) \|_{\mathbf{H}^{m}}\right.\right. \\
& \quad \leq C\left\|\Phi\left(\overline{\eta \psi^{+}}, \eta \psi^{+}, \overline{\delta \psi^{+}}, \delta \psi^{+}\right)\right\|_{\mathcal{X}} \\
& \quad \leq C\left(\left\|\overline{\omega_{1, c}}\right\|_{\mathbf{H}^{m}}^{2}+\left\|\omega_{1, c}\right\|_{\mathbf{H}^{m}}^{2}+\left\|\overline{v_{1, c}}\right\|_{\mathbf{H}^{m}}^{2}+\left\|v_{1, c}\right\|_{\mathbf{H}^{m}}^{2}\right) \\
& \quad \leq C\left(\left\|\eta \psi^{+}\right\|_{\mathbf{H}^{m}}^{2}+\left\|\delta \psi^{+}\right\|_{\mathbf{H}^{m}}^{2}\right) \leq C\left(|\eta|^{2}+|\delta|^{2}\right),
\end{aligned}
$$

where we use the notation $(\widetilde{\omega}, \widetilde{v})=\Phi\left(\omega_{c}, v_{c}\right)=\Phi\left(\overline{\eta \psi^{+}}, \eta \psi^{+}, \overline{\delta \psi^{+}}, \delta \psi^{+}\right)$. Inspired by the classical Hopf-bifurcation result [16], one can employ the implicit function 
theorem to find real value solutions (i.e. find $\left.\left(\gamma_{1}, \gamma_{2}\right)=(\eta, \delta) \in \mathbf{R}^{2}\right)$ of equations (3.39)-(3.40). Hence, we define the complex-valued smooth function

$$
\begin{aligned}
& \Upsilon^{1}\left(\gamma_{1}, \gamma_{2} ; \varrho, \beta\right):= \begin{cases}-i\left(\xi_{0}+\varrho\right)+\lambda_{0}^{+}\left(\beta_{c}+\varepsilon\right)+\gamma_{1}^{-1} g^{3}\left(\beta_{c}+\epsilon, \gamma_{1}, \gamma_{2}\right), & \gamma_{1} \neq 0, \\
-i\left(\xi_{0}+\varrho\right)+\lambda_{0}^{+}\left(\beta_{c}+\varepsilon\right), & \gamma_{1}=0,\end{cases} \\
& \Upsilon^{2}\left(\gamma_{1}, \gamma_{2} ; \varrho, \beta\right):= \begin{cases}-i\left(\xi_{0}+\varrho\right)+\mu_{0}^{+}\left(\beta_{c}+\varepsilon\right)+\gamma_{2}^{-1} g^{4}\left(\beta_{c}+\epsilon, \gamma_{1}, \gamma_{2}\right), & \gamma_{2} \neq 0, \\
-i\left(\xi_{0}+\varrho\right)+\mu_{0}^{+}\left(\beta_{c}+\varepsilon\right), & \gamma_{2}=0 .\end{cases}
\end{aligned}
$$

It follows from $\left(\lambda_{0}^{+}\left(\beta_{c}\right), \mu_{0}^{+}\left(\beta_{c}\right)\right)=\left(i \xi_{0}, i \xi_{0}\right)$ that $\left(\Upsilon^{1}(0,0,0,0), \Upsilon^{2}(0,0,0,0)\right)=$ $(0,0)$. Moreover, by assumption (H2) the Jacobi matrix

$$
\begin{aligned}
& \left.\mathbf{D}_{\rho, \varepsilon} \Upsilon^{1}\left(\gamma_{1}, \gamma_{2} ; \varrho, \varepsilon\right)\right|_{\gamma_{1}=\gamma_{2}=\varrho=\varepsilon=0}=\left(\begin{array}{cc}
0 & \left.\frac{d}{d \beta} \operatorname{Re} \lambda_{0}^{+}(\beta)\right|_{\beta=\beta_{c}} \\
-1 & \left.\frac{d}{d \beta} \operatorname{Im} \lambda_{0}^{+}(\beta)\right|_{\beta=\beta_{c}}
\end{array}\right), \\
& \left.\mathbf{D}_{\rho, \varepsilon} \Upsilon^{2}\left(\gamma_{1}, \gamma_{2} ; \varrho, \varepsilon\right)\right|_{\gamma_{1}=\gamma_{2}=\varrho=\varepsilon=0}=\left(\begin{array}{cc}
0 & \left.\frac{d}{d \beta} \operatorname{Re} \mu_{0}^{+}(\beta)\right|_{\beta=\beta_{c}} \\
-1 & \left.\frac{d}{d \beta} \operatorname{Im} \mu_{0}^{+}(\beta)\right|_{\beta=\beta_{c}}
\end{array}\right),
\end{aligned}
$$

with respect to $\rho, \varepsilon$ has

$$
\begin{gathered}
\left.\operatorname{det} \mathbf{D}_{\rho, \varepsilon} \Upsilon^{1}\left(\gamma_{1}, \gamma_{2} ; \varrho, \varepsilon\right)\right|_{\gamma_{1}=\gamma_{2}=\varrho=\varepsilon=0}=\left.\frac{d}{d \beta} \operatorname{Re} \lambda_{0}^{+}(\beta)\right|_{\beta=\beta_{c}}>0, \\
\left.\operatorname{det} \mathbf{D}_{\rho, \varepsilon} \Upsilon^{2}\left(\gamma, \gamma_{2} ; \varrho, \varepsilon\right)\right|_{\gamma_{1}=\gamma_{2}=\varrho=\varepsilon=0}=\left.\frac{d}{d \beta} \operatorname{Re} \mu_{0}^{+}(\beta)\right|_{\beta=\beta_{c}}>0 .
\end{gathered}
$$

Thus, for sufficient small $\gamma_{1}, \gamma_{2}>0$, we find a function $\gamma_{1} \mapsto\left(\varrho\left(\gamma_{1}\right), \varepsilon\left(\gamma_{1}\right)\right)$ and $\gamma_{2} \mapsto\left(\varrho\left(\gamma_{2}\right), \varepsilon\left(\gamma_{2}\right)\right)$ with $\varrho(0)=\varepsilon(0)=0$ such that

$$
\begin{aligned}
& -i\left(\xi_{0}+\varrho\left(\gamma_{1}\right)\right)+\lambda_{0}^{+}\left(\beta_{c}+\varepsilon\left(\gamma_{1}\right)\right)-\gamma_{1}^{-1} g^{3}\left(\beta_{c}+\varepsilon\left(\gamma_{1}\right), \gamma_{1}, \beta_{c}+\varepsilon\left(\gamma_{2}\right), \gamma_{2}\right)=0, \\
& -i\left(\xi_{0}+\varrho\left(\gamma_{2}\right)\right)+\mu_{0}^{+}\left(\beta_{c}+\varepsilon\left(\gamma_{2}\right)\right)-\gamma_{2}^{-1} g^{4}\left(\beta_{c}+\varepsilon\left(\gamma_{1}\right), \gamma_{1}, \beta_{c}+\varepsilon\left(\gamma_{2}\right), \gamma_{2}\right)=0 .
\end{aligned}
$$

Note that the degree of nonlinearity. Then it follows from differentiating this equation that $\varepsilon^{i} \neq 0$ for some first $i$. Hence, the function $\gamma_{1} \mapsto \varepsilon\left(\gamma_{1}\right)$ and $\gamma_{1} \mapsto \varepsilon\left(\gamma_{2}\right)$ are locally invertible, and have $\varepsilon \mapsto \gamma_{1}(\varepsilon)$ and $\varepsilon \mapsto \gamma_{2}(\varepsilon)$. It implies that the following equation holds

$$
\begin{aligned}
& -i\left(\xi_{0}+\varrho\left(\gamma_{1}(\varepsilon)\right)\right) \gamma_{1}(\varepsilon)+\lambda_{0}^{+}\left(\beta_{c}+\varepsilon\right) \gamma_{1}(\varepsilon)-g^{3}\left(\beta_{c}+\varepsilon, \gamma_{1}(\varepsilon), \gamma_{2}(\varepsilon)\right)=0, \\
& -i\left(\xi_{0}+\varrho\left(\gamma_{2}(\varepsilon)\right)\right) \gamma_{2}(\varepsilon)+\mu_{0}^{+}\left(\beta_{c}+\epsilon\right) \gamma_{2}(\varepsilon)-g^{4}\left(\beta_{c}+\varepsilon, \gamma_{1}(\varepsilon), \gamma_{2}(\varepsilon)\right)=0,
\end{aligned}
$$

for sufficient small $\varepsilon>0$.

Therefore we obtain the desired solutions of (3.35)-(3.36) by setting

$$
\left(\xi_{1}, \xi_{2}\right)=\left(\xi_{0}+\varrho\left(\gamma_{1}(\varepsilon)\right), \xi_{0}+\varrho\left(\gamma_{2}(\varepsilon)\right)\right), \quad \beta=\beta_{c}+\varepsilon,
$$




$$
\left(\omega_{1, c}, v_{1, c}\right)=\left(\gamma_{1}(\varepsilon) \psi_{\beta_{c}+\varepsilon}^{+}, \gamma_{2}(\varepsilon) \psi_{\beta_{c}+\varepsilon}^{+}\right)(x) .
$$

This result combines with Lemma 2.5 giving the proof of Theorem 1.1.

\section{Proof of Theorem 1.2}

In this section, inspired by the work [5], we will use Bootstrap Techniques to prove that linear stability of smooth time periodic solution implies nonlinear stability for MHD in $\mathcal{L}^{p}$ for $p>3$.

Assume that $\left(U_{\alpha}, H_{\alpha}\right)$ is the smooth periodic solution of (1.1)-(1.3) and $c_{1}=0 . \mathbf{P}$ denotes the Leray projection onto the space of divergence free functions. We introduce the deviation

$$
u=U-U_{\alpha}, \quad v=H-H_{\alpha} .
$$

Then, we can obtain:

$$
\begin{aligned}
u_{t} & =A u+N_{1}(u, v), \\
v_{t} & =B v^{\prime}+N_{2}(u, v), \\
\left.u\right|_{t=0} & =u_{0},\left.\quad v\right|_{t=0}=v_{0},
\end{aligned}
$$

where

$$
\begin{aligned}
A u & =\mathbf{P}\left[\nu \triangle u-u_{\alpha} \cdot \nabla u-u \cdot \nabla u_{\alpha}-H_{\alpha} \cdot \nabla u+H_{\alpha} \cdot \nabla u+u \cdot \nabla H_{\alpha}\right] \\
B v^{\prime} & =\mathbf{P}\left[\eta \triangle v-u_{\alpha} \cdot \nabla v-u \cdot \nabla v_{\alpha}+v_{\alpha} \cdot \nabla u+v \cdot \nabla u_{\alpha}\right]
\end{aligned}
$$

and

$$
\begin{aligned}
& N_{1}(u, v)=\mathbf{P}\left[-\nabla \cdot(u \otimes u)-\frac{1}{2} \nabla|v|^{2}+\nabla \cdot(v \otimes v)\right], \\
& N_{2}(u, v)=\mathbf{P}[-\nabla \cdot(u \otimes v)+\nabla \cdot(v \otimes u)] .
\end{aligned}
$$

By [22], we note that the linear periodic operator $A$ and $B$ is a bounded perturbation of the Stokes operator $\mathbf{P} \triangle$. The operator $A$ and $B$ generates a strongly continuous semigroup in every Sobolev space $\mathbf{W}^{s, p}$ which we denote by $e^{A t}$ and $e^{B t}$ :

$$
\begin{array}{ll}
u(t)=e^{A t} u_{0}, & u_{0} \in \mathbf{W}^{s, p}, \\
v(t)=e^{B t} v_{0}, & v_{0} \in \mathbf{W}^{s, p} .
\end{array}
$$

Let $\lambda_{1}$ and $\lambda_{2}$ be the eigenvalue of $A$ and $B$ with maximal positive real part, which we denote by $\mu_{1}$ and $\mu$, and let $\phi_{1}, \phi_{2} \in \mathbf{L}^{p}$, with $\|\phi\|_{\mathbf{L}^{p}}=\|\phi\|_{\mathbf{L}^{p}}=1$, be the corresponding eigenfunction. For fixed $0<\sigma_{1}<\lambda_{1}$ and $0<\sigma_{2}<\lambda_{2}$ we denote by $A_{\sigma}$ and $B_{\sigma}$ the following operator:

$$
A_{\sigma_{1}}=A-\lambda_{1}-\sigma_{1}, \quad B_{\sigma_{2}}=B-\lambda_{2}-\sigma_{2} .
$$

Let us fix an arbitrary small $\varepsilon>0$, and solve the Cauchy problem (4.1)-(4.2) with initial condition $\left(u_{0}, v_{0}\right)=\left(\varepsilon \phi_{1}, \varepsilon \phi_{2}\right)$. By [23], we note that for such initial condition, with $\varepsilon$ small enough, there exists a unique global in time classical 
solution to (4.1)-(4.2). Using Duhamel's formula we write the solution in the form

$$
\begin{aligned}
& u_{t}=u_{0} e^{\lambda_{1} t}+\int_{0}^{t} e^{A(t-s)} N_{1}(u, v)(s) d s, \\
& v_{t}=v_{0} e^{\lambda_{2} t}+\int_{0}^{t} e^{B(t-s)} N_{2}(u, v)(s) d s .
\end{aligned}
$$

Before we show the proof of Theorem 1.3, we state the following Sobolev embedding theorem which is taken from [5].

Lemma 4.1. Let $s>0,1<r_{1}<\infty$ and $1<r_{2}<\infty$ satisfy

$$
\frac{1}{r_{1}}<1-\frac{s}{n}, \quad r_{2} \leq r_{1}, \quad \frac{1}{r_{2}} \leq \frac{1}{r_{1}}+\frac{s}{n} .
$$

Then $\|f\|_{W^{-s, r_{1}}} \leq\|f\|_{L^{r_{2}}}$.

Proof of Theorem 1.3. By assumption (H3), the spectrum of linear time periodic operators $A$ and $B$ are strictly bounded away from the imaginary axis in the left half plane for all $\alpha \in\left[\alpha_{c}-\alpha_{0}, \alpha_{c}+\alpha_{0}\right]$. So there exist positive constants $\mu_{1}$ and $\mu_{2}$ such that

$$
\left\|e^{A t} u_{0}\right\|_{\mathbf{L}^{q}} \leq C e^{-\mu_{1} t}\left\|u_{0}\right\|_{\mathbf{L}^{q}}, \quad\left\|e^{B t} v_{0}\right\|_{\mathbf{L}^{q}} \leq C e^{-\mu_{2} t}\left\|v_{0}\right\|_{\mathbf{L}^{q}},
$$

for all $t>0$ and $\left(u_{0}, v_{0}\right) \in \mathcal{L}^{p}$.

Then by (4.7)-(4.8) and Lemma 2.4, we derive

$$
\begin{aligned}
\|u\|_{\mathbf{L}^{q}} & =\left\|e^{A t} u_{0}\right\|_{\mathbf{L}^{q}}+\left\|\int_{0}^{t} e^{-\mu_{1}(t-s)} A^{\gamma} e^{A(t-s)} A^{-\gamma} N_{1}(u, v)(s) d s\right\|_{\mathbf{L}^{q}} \\
& \leq C e^{-\mu_{1} t}\left\|u_{0}\right\|_{\mathbf{L}^{q}}+C \int_{0}^{t} e^{-\mu_{1}(t-s)} \frac{1}{(t-s)^{\gamma}}\left\|A^{-\gamma} N_{1}(u, v)(s)\right\|_{\mathbf{L}^{q}} d s,
\end{aligned}
$$

and

$$
\text { (4.10) } \begin{aligned}
\|v\|_{\mathbf{L}^{q}} & =\left\|e^{B t} v_{0}\right\|_{\mathbf{L}^{q}}+\left\|\int_{0}^{t} e^{-\mu_{2}(t-s)} A^{\gamma} e^{B(t-s)} B^{-\gamma} N_{2}(u, v)(s) d s\right\|_{\mathbf{L}^{q}} \\
& \leq C e^{-\mu_{2} t}\left\|v_{0}\right\|_{\mathbf{L}^{q}}+C \int_{0}^{t} e^{-\mu_{2}(t-s)} \frac{1}{(t-s)^{\gamma}}\left\|B^{-\gamma} N_{2}(u, v)(s)\right\|_{\mathbf{L}^{q}} d s .
\end{aligned}
$$

Then, by Lemma 2.5, (4.5)-(4.6) and the continuity of the Leray projection, we have

$$
\begin{aligned}
\left\|A^{-\alpha} N_{1}(u, v)(s)\right\|_{\mathbf{L}^{q}} & \leq\left\|N_{1}(u, v)(s)\right\|_{\mathbf{W}^{-2 \gamma, q}} \leq\left\|N_{1}(u, v)(s)\right\|_{\mathbf{W}^{-2 \gamma, q}} \\
& \leq C\left(\|u \otimes u\|_{\mathbf{W}^{1-2 \gamma, q}}+\|v\|_{\mathbf{W}^{1-2 \gamma, q}}^{2}+\|v \otimes v\|_{\mathbf{W}^{1-2 \gamma, q}}\right),
\end{aligned}
$$

and

$$
\begin{aligned}
\left\|B^{-\alpha} N_{2}(u, v)(s)\right\|_{\mathbf{L}^{q}} & \leq\left\|N_{2}(u, v)(s)\right\|_{\mathbf{W}^{-2 \gamma, q}} \leq\left\|N_{2}(u, v)(s)\right\|_{\mathbf{W}^{-2 \gamma, q}} \\
& \leq C\left(\|u \otimes v\|_{\mathbf{W}^{1-2 \gamma, q}}+\|v \otimes u\|_{\mathbf{W}^{1-2 \gamma, q}}\right) .
\end{aligned}
$$


We choose $\gamma$ close to 1 so that $q>3 /(2 \gamma-1)$. This means that the conditions in Lemma 4.1 is satisfied with $s=2 \gamma-1, r_{1}=q$ and $r_{2}=q / 2$. Thus, we have

$$
\begin{aligned}
\left\|A^{-\gamma} N_{1}(u, v)(s)\right\|_{\mathbf{L}^{q}} & \leq C\left(\|u \otimes u\|_{\mathbf{L}^{q / 2}}+\|v\|_{\mathbf{L}^{q / 2}}^{2}+\|v \otimes v\|_{\mathbf{L}^{q / 2}}\right) \\
& \leq C\left(\|u\|_{\mathbf{L}^{q}}^{2}+\|v\|_{\mathbf{L}^{q}}^{2}\right) \\
\left\|B^{-\gamma} N_{2}(u, v)(s)\right\|_{\mathbf{L}^{q}} & \leq C\left(\|u \otimes v\|_{\mathbf{L}^{q / 2}}+\|v \otimes u\|_{\mathbf{L}^{q / 2}}\right) \leq C\|u\|_{\mathbf{L}^{q}}\|v\|_{\mathbf{L}^{q}} .
\end{aligned}
$$

Thus, by (4.9)-(4.10), we obtain

$$
\begin{aligned}
\|u\|_{\mathbf{L}^{q}} \leq & C e^{-\mu_{1} t}\left\|u_{0}\right\|_{\mathbf{L}^{q}} \\
& +C \int_{0}^{t} e^{-\mu_{1}(t-s)} \frac{1}{(t-s)^{\gamma}}\left(\|u(s)\|_{\mathbf{L}^{q}}^{2}+\|v(s)\|_{\mathbf{L}^{q}}^{2}\right) d s,
\end{aligned}
$$

and

(4.13) $\|v\|_{\mathbf{L}^{q}} \leq C e^{-\mu_{2} t}\left\|v_{0}\right\|_{\mathbf{L}^{q}}+C \int_{0}^{t} e^{-\mu_{2}(t-s)} \frac{1}{(t-s)^{\gamma}}\|u(s)\|_{\mathbf{L}^{q}}\|v(s)\|_{\mathbf{L}^{q}} d s$.

Let $T$ be the maximal time for which

$$
\begin{aligned}
\|u\|_{\mathbf{L}^{q}} \leq 2 C e^{-\mu_{1} t}\left\|u_{0}\right\|_{\mathbf{L}^{q}}, & t \leq T, \\
\|v\|_{\mathbf{L}^{q}} \leq 2 C e^{-\mu_{2} t}\left\|v_{0}\right\|_{\mathbf{L}^{q}}, & t \leq T,
\end{aligned}
$$

Combining (4.11)-(4.15), we have

$$
\begin{aligned}
\|v\|_{\mathbf{L}^{q}} & \leq C e^{-\mu_{2} t}\left\|v_{0}\right\|_{\mathbf{L}^{q}}\left(1+4 C^{2}\left\|u_{0}\right\|_{\mathbf{L}^{q}} e^{-\mu_{1} t}\right) \\
& \leq C e^{-\mu_{2} t}\left\|v_{0}\right\|_{\mathbf{L}^{q}}\left(1+4 C^{2}\left\|u_{0}\right\|_{\mathbf{L}^{q}}\right), \\
\|u\|_{\mathbf{L}^{q}} & \leq C e^{-\mu_{1} t}\left\|u_{0}\right\|_{\mathbf{L}^{q}}\left(1+4 C^{2}\left\|u_{0}\right\|_{\mathbf{L}^{q}} e^{-\mu_{1} t}\right)+4 C^{3}\left\|v_{0}\right\|_{\mathbf{L}^{q}}^{2} e^{-2 \mu_{2} t},
\end{aligned}
$$

for $t \leq T$. Summing up (4.16)-(4.17), we have

(4.18) $\|u\|_{\mathbf{L}^{q}}+\|v\|_{\mathbf{L}^{q}} \leq C e^{-\mu_{1} t}\left\|u_{0}\right\|_{\mathbf{L}^{q}}\left(1+4 C^{2}\left\|u_{0}\right\|_{\left.\mathbf{L}^{q} e^{-\mu_{1} t}\right)}\right.$

$$
+4 C^{3}\left\|v_{0}\right\|_{\mathbf{L}^{q}}^{2} e^{-2 \mu_{2} t}+C e^{-\mu_{2} t}\left\|v_{0}\right\|_{\mathbf{L}^{q}}\left(1+4 C^{2}\left\|u_{0}\right\|_{\mathbf{L}^{q}}\right),
$$

for $t \leq T$. We choose $\left\|u_{0}\right\|_{\mathbf{L}^{q}}<1 /\left(8 C^{2}\right),\left\|v_{0}\right\|_{\mathbf{L}^{q}} \leq 1 /\left(16 C^{2}\right)$. Then, by (4.18), we obtain

$$
\|u\|_{\mathbf{L}^{q}}+\|v\|_{\mathbf{L}^{q}} \leq \frac{3 C}{2} e^{-\mu_{1} t}\left\|u_{0}\right\|_{\mathbf{L}^{q}}+\frac{7 C}{4} e^{-\mu_{2} t}\left\|v_{0}\right\|_{\mathbf{L}^{q}}, \quad t \leq T
$$

But from (4.14)-(4.15), we have

$$
\|u\|_{\mathbf{L}^{q}}+\|v\|_{\mathbf{L}^{q}} \leq 2 C\left(e^{-\mu_{1} t}\left\|u_{0}\right\|_{\mathbf{L}^{q}}+e^{-\mu_{2} t}\left\|v_{0}\right\|_{\mathbf{L}^{q}}\right), \quad t \leq T .
$$

Therefore, (4.20) implies the smaller bound of (4.19), which means a contradiction with a maximal time $T$. Thus, $T=\infty$ and the bound (4.20) holds for all $t \geq 0$. 
Acknowledgements. The author expresses his sincere thanks to the anonymous three referees for very careful reading and for providing many valuable comments and suggestions which led to improvement of this paper. This work is supported by NSFC No 11201172, SRFDP Grant No 20120061120002 and the Fundamental Research Funds for the Central Universities of Xiamen University, No 20720150013.

\section{REFERENCES}

[1] T. Brand, M. Kunze, G. Schneider and T. Seelbach, Hopf bifurcation and exchange of stability in diffusive media, Arch. Rational Mech. Anal. 171 (2004), 263-296.

[2] Z.M. Chen And W.G. Price, it Time dependent periodic Navier-Stokes flows on a twodimensional torus, Comm. Math. Phys 179 (1996), 577-597.

[3] B. Climent-Ezquerra, F. Guillen-Gonzalez and M.A. Rojas-Medar, Time-periodic solutions for a generalized Boussinesq model with Neumann boundary conditions for temperature, Proc. Roy. Soc. Sect. A 463 (2007), 2153-2164.

[4] M.G. Crandall and P. Rabinowitz, The Hopf Bifurcation theorem in infinite dimensions, Arch. Rational Mech. Anal. 67 (1977), 53-72.

[5] S. Friendlander, N. Pavlović and R. Shvydkoy, Nonlinear instability for the NavierStokes equations, Commun. Math. Phys. 264 (2006), 335-347.

[6] S. Friendlander, W. Strauss and M. Vishik, Nonlinear instability in an ideal fluids, Ann. Inst. H. Poincaré Anal. Non Linéaire 14 (1997), 187-209.

[7] Y. Giga, Analyticity of the semigroup generated by the Stokes operator in $\boldsymbol{L}_{r}$ spaces, Math. Z. 178 (1981), 297-329.

[8] — The Stokes operator in $\boldsymbol{L}_{r}$ spaces, Proc. Japan Acad. Ser. A Math. Sci. 57 (1981), $85-89$.

[9] R. Glowinski and G. Guidoboni, Hopf bifurcation in viscous incompressible flow down an inclined plane: a numerical approach, J. Math. Fluid Mech. 10 (2008), 434-454.

[10] D. Henry, Geometric Theory of Semilinear Parabolic Equations, Springer-Verlag, New York, 1981.

[11] G. Iooss, Bifurcation des solutions périodiques de certains problèmes dévolution, C.R. Acad. Sci. Paris Sér. A 273 (1971), 624-627.

[12] _ Bifurcation of a periodic solution of the Navier-Stokes equations into an invariant torus, Arch. Rational Mech. Anal. 58 (1975), 35-56.

[13] G. Iooss AND A. Mielke, Bifurcating time-periodic solutions of Navier-Stokes equations in infinite cylinders, J. Nonlinear Sci. 1 (1991), 107-146.

[14] V.I. Iudovich, Apprearance of auto-oscillations in a fluid, Prikl. Mat. Mekh. 35 (1971), 638-655.

[15] J. LERAY, Essai sur le mouvement d'un liquide visqueux emplissant l'espace, Acta Math. 63 (1934), 193-248.

[16] J.E. Marsden and M. Mccracken, The Hopf Bifurcation and its Applications, Springer, Berlin, New York, 1976

[17] A. Melcher, G. Schneider and H. Uecker, A Hopf-bifurcation theorem for the vorticity formulation of the Navier-Stokes equations in $\mathbb{R}^{3}$, Commun. Partial Differential Equations 33 (2008), 772-783.

[18] A. PAZY, Semigroups of Linear Operators and Applications to Partial Differential Equations, Springer-Verlag, New York, 1983. 
[19] D.H. Sattinger, Bifurcation of periodic solutions of the Navier-Stokes equations, Arch. Rational Mech. Anal. 41 (1971), 66-80.

[20] V.A. Solonnikov, Some stationary boundary value problems of magnetohydrodynamic, Trudy Mat. Inst. Steklov. 59 (1960), 174-187.

[21] M. Vishik AND S. Friendlander, Nonlinear instability in two dimensional ideal fluids: the case of a dominant eigenvalue, Commun. Math. Phys. 243 (2003), 261-273.

[22] V. I. Yudovich, The Linearization Method in Hydrodynamical Stability Theory, Transl. Math. Monogr. vol. 74, Providence, RI, Amer. Math. Soc. 1989.

[23] R. Temam, Some Developments on Navier-Stokes Equations in the Second Half of the 20th Century. Development of Mathematics 1950-2000, Basel, Birkhäuser, 2000, 10491106.

Manuscript received January 9, 2014

WEIPING YAN

School of Mathematical Sciences

Xiamen University

Xiamen 361005, P.R. CHINA

E-mail address: yan8441@126.com 